\title{
1 Strain rate dependent plasticity and fracture of DP1000 2 steel under proportional and non-proportional loading
}

3

4 Sarath Chandran ${ }^{\mathrm{a}^{*}}$, Wenqi Liu ${ }^{\mathrm{b}, \mathrm{c}}$, Junhe Lian $^{\mathrm{b}}$, Sebastian Münstermann ${ }^{\mathrm{c}}$, Patricia Verleysen $^{\mathrm{a}}$

5 a MST-DyMaLab, Department of Electromechanical, Systems and Metal Engineering, Ghent

6 University, Technologiepark 46, 9052, Zwijnaarde, Belgium

$7{ }^{\mathrm{b}}$ Advanced Manufacturing and Materials, Department of Mechanical Engineering, Aalto

8 University, Puumiehenkuja 3, 02150, Espoo, Finland

$9{ }^{c}$ Integrity of Materials and Structures, Steel Institute, RWTH Aachen University, Intzestraße 10 1, 52072, Aachen, Germany

11

* Corresponding author

Email address : Sarath.Chandran@UGent.be

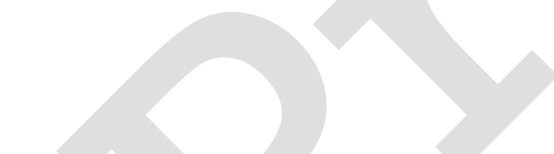

20 
Abstract

To assess the effect of stress state and strain rate on damage and fracture of a commercial DP1000 steel with a very fine microstructure, an extensive series of tests were performed. Using finite element simulations, eight different sample geometries, including a dogbone, a central hole, a shear and several notched samples, were designed to achieve both proportional and nonproportional stress states using conventional test benches. Tested at quasi-static, intermediate and, dynamic deformation rates, in total 175 tests were performed. Local strain fields were obtained by digital image correlation. A correction procedure was worked out to eliminate the influence of thermal softening. After testing, scanning electron microscopy was employed to analyse the fracture surfaces. Tests and fractography allowed to draw systematic conclusions on the response of the DP1000 steel. A two-stage strain rate sensitivity of strength is found with a gradually increasing slope at low strain rates and a much steeper rise at high strain rates, which is further amplified at higher triaxiality stress states. The experimentally derived fracture loci revealed a dominant, detrimental impact of the stress triaxiality that is most pronounced at intermediate strain rates. A remarkable, non-monotonic evolution of the fracture strain with strain rate is observed: the highest values were obtained at intermediate rates. Scanning electron microscopy images of the fracture surfaces indicate a void-assisted ductile fracture, though with the occurrence of brittle features triggered at dynamic strain rates. Fracture morphology and dimple features are heavily dependent on stress state, strain rate and loading path.

\section{Keywords}

Ductile fracture, impact testing, multiaxial stress state, ductility, strain rate behaviour, dualphase steel, fracture mechanisms 
1. Design of test campaign imposing proportional and non-proportional stress paths

51

52

53

54

55

56

2. Extensive test dataset on strain rate dependent plasticity and fracture of DP1000

3. DP1000 shows a positive, two-stage strain rate sensitivity of strength

4. Remarkable, non-monotonic evolution of fracture strain of DP1000 with strain rate

5. Pronounced stress state influence on DP1000 hardening, ductility and failure modes 


\section{Introduction}

The advent of advanced high strength steels (AHSS) has succeeded in reconciling the often conflicting demands of enhanced safety and reduced fuel consumption in the automotive industry. Among AHSS, dual-phase (DP) steels have a great potential for vehicle body design [ULSAB, 2016], particularly, for crash applications due to their excellent combination of strength, initial work hardening and ductility.

The mechanical properties of DP steels are the result of a multiphase microstructure consisting of hard martensite islands, or in some cases, bainite with traces of retained austenite, spatially dispersed in a soft ferritic matrix. The ferritic phase lies at the origin of the excellent ductility, whereas the high strength is mainly provided by the martensite. However, the presence of different microstructural constituents and their inevitable interaction, introduce another level of complexity to damage and fracture of multiphase steels [Lian, 2015]. The fairly straightforward sequence of plastic deformation, severe strain localization and ductile fracture observed for common single-phase ductile steels may no longer be applicable. Premature failure of multiphase steels during forming processes or in service can restrict the applicability of multiphase steels and should therefore be accounted for. Differences in mechanical response under dynamic loading, as compared to quasi-static loading, substantiate the need to explore the intrinsic dynamic nature of the damage and fracture phenomena in multiphase automotive steels.

The effect of strain rate on the mechanical properties of metal alloys, including DP steels, has been extensively studied [Chen et al., 2011; Singh et al., 2011; Kim et al., 2013; Qin et al., 2013; Anderson et al., 2014; Rahman et al., 2016, Erice et al., 2018]. Several studies reported a positive effect of strain rate on the strength and strain hardening of dual-phase steels [Beynon et al., 2005; Oliver et al., 2007; Yu et al., 2009; Chen et al., 2011; Singh et al., 2011; Qin et al., 2013; Kim et al., 2013; Anderson et al., 2014; Cao et al., 2015; Rahman et al., 2016; Erice 
et al., 2018]. However, significantly different strain rate dependencies of the flow stress were observed in DP steels with strength levels ranging from 600 to $1000 \mathrm{MPa}$ attributed to changing martensite phase fractions [Kim et al., 2013; Wang et al., 2013; Rahman et al. 2016, Das et al., 2017; Xu et al., 2019]. The strain rate effect on ductility, however, led to contradictory conclusions [Yu et al., 2009; Wang et al., 2013; Alturk et al., 2017]. The studies demonstrate the uniqueness of the microstructure-dynamic property relations in multiphase high strength steels and accentuate the need for in-depth characterization in light of their reliable utilization in various sectors.

Extrinsic factors such as imposed strain, stress state, temperature and strain rate affect damage and fracture in DP steels next to the material morphological features. Concerning the stress state, the stress triaxiality and Lode angle are the critical factors that govern ductile fracture in metals [Bao and Wierzbicki, 2004; Bai and Wierzbicki, 2008]. Indeed, depending on the stress state imparted on the material, the dominant fracture mode can alternate between void-assisted tensile or shear failure and a combined shear-tensile failure. A wide variety of damage mechanisms can thus be unlocked by introducing various stress states. These states can be achieved by imposing different loads or load combinations on well-designed samples [Bao and Wierzbicki, 2004; Mohr and Doyoyo, 2004; Mohr and Henn, 2007; Roth and Mohr 2014]. However, very often, the reliability of the damage and fracture assessment is detrimentally affected by the complex design of purpose-developed test benches and the critical manufacturing of the complicated samples geometries. More recently, specimen geometries have been designed imposing specific stress states on metal samples using universal tensile machines [Bao and Wierzbicki; 2004; Mohr and Doyoyo, 2004; Mohr and Henn, 2007; Driemer et al., 2010; Peirs et al., 2012; Roth and Mohr, 2014]. Damage and fracture investigations on DP steels [Steinbrunner et al., 1988; Mazinani and Poole, 2007; Kadkhodapour et al., 2011; Landron et al., 2013; Ghadbeigi et al., 2013; Anderson et al., 2014; 
Erice et al., 2018; Chiyatan and Uthaisangsuk, 2020] revealed a negative impact of stress triaxiality on plastic strain to failure while the strain rate yields a positive response in some steel grades. Certain studies considered, next to stress triaxiality, also the Lode angle and represented the stress state dependency on fracture initiation and failure strain by a three-dimensional locus [Bai and Wierzbicki, 2008; Lian et al., 2013; Roth and Mohr, 2014; Wu et al., 2017; Erice at al., 2018; Roth and Mohr, 2018] using finite element simulations. A ductile mode of failure was generally observed in DP steels. Seemingly divergent void nucleation mechanisms revealed the complexity, making a clear understanding and prediction of the damage behaviour complicated. Upon critical review of studies concerning ductile fracture, it is evident that the vast majority of material specimens undergoes a continuous variation in loading and/or strain path during the deformation process. The stress and strain path changes result in a nonstraightforward assessment of material damage and fracture, including the fracture locus. Moreover, non-proportional loading paths limit the direct correlation between stress state and observed microstructural damage mechanisms. Hence, the establishment of proportional loading paths in experimental design is crucial for the unambiguous analysis of damage and fracture.

The present study aims at an in-depth characterisation of the plasticity, damage and fracture of a commercial DP1000 steel with an ultra-fine grain microstructure. To trigger the multitude of damage mechanisms typically met in crash energy absorption components, an extensive test campaign was designed aiming at a wide range of stress states and strain rates. Eight carefully designed sample geometries were tested in uniaxial test setups. Deliberately, both proportional and non-proportional stress paths were imposed. Indeed, although proportional loading states facilitate systematic analysis and interpretation of the test result, in real car components, proportional loading states are quite exceptional. All samples were tested at several, 7 in total, quasi-static, intermediate and high strain rate tests using a conventional tensile test machine and 
a split Hopkinson bar tensile setup. Including repetitions and tests performed for a temperature correction, 175 tests were carried out.

Several advanced measurement techniques were used during all tests, including static and dynamic 3D digital image correlation to obtain full-field strain fields. Additional temperature measurements allowed to accurately determine the Taylor-Quinney coefficient, i.e., the fraction of plastic work converted into heat. An experimental strategy was devised to uncouple the effect of strain rate and temperature on the plastic response of the studied steel. To identify and distinguish the various damage and fracture modes prevalent in the DP1000 steel, fracture surfaces of the samples were analysed using scanning electron microscopy (SEM).

Finite element simulations were used for the specimen geometry optimization and to support test analysis. The desired stress states are attained by well-conceived, easy-to-manufacture sample geometries tested using widely available experimental setups and measurement techniques.

\section{Material and Methods}

\subsection{Material}

A commercial dual-phase steel (DP1000) in the form of a rolled sheet with a thickness of 1.5 $\mathrm{mm}$ is used in this investigation. The microstructure consists of a mixture of ferrite grains (55\%) and martensite islands (45\%). The average grain size of the ferrite is less than $2 \mu \mathrm{m}$.

\subsection{Experimental methods}

\subsubsection{Test setups, measurements and data processing}

The static and intermediate strain rate tests are performed on an Instron material testing machine (Model 5569). The deformation rate is controlled by the speed of the cross-head. A calibrated load cell is used to measure the force. 
An optical full-field surface deformation measurement technique based on digital image correlation (DIC) using the commercial MatchID software is adopted to monitor the evolution of the sample surface strain during testing. To this purpose, prior to testing, a speckle pattern is applied to the sample which is recorded during deformation by two cameras positioned under an angle $\left(30-40^{\circ}\right)$. High-resolution images are obtained using 5Mpxl F-033B Allied Vision Stingray cameras together with appropriate LED lighting. The setup is calibrated to accurately quantify 3D, i.e., both in-plane and out of plane, displacements.

The split Hopkinson bar tensile (SHBT) setup of DyMaLab at Ghent University is used for the high strain rate tests. In the setup, the sample is positioned between two long bars, i.e., the input and output bar. A tensile loading wave is generated by the direct impact of an impactor on the free end of the input bar. The incident loading wave propagates towards the sample and is partly reflected and partly transmitted. By controlling the velocity of the impactor, the strain rate in the sample can be adjusted. Strain gauge measurements on the bars of the waves allow to calculate the force, relative velocity and displacement imposed on the sample/bar interfaces [Verleysen et al., 2008]. Also for the dynamic tests, 3D DIC is used for full-field strain measurements. The applied speckle pattern is recorded during the tests by two high speed Photron Mini AX200 cameras. Depending on the test, framerates between 50,000 and 100,000 fps are used. The sample surface is illuminated using a high-intensity Dedocool light set.

To ensure the statistical relevance of the results, for each test condition, three test repetitions are performed. For both static and dynamic DIC processing, subset, step and strain window sizes are adopted based on the average speckle size $(25-55 \mu \mathrm{m})$ and resolution $(5-10 \mu \mathrm{m} / \mathrm{pixel})$. The following strain formulations are used in the result section. For uniaxial tensile tests, often the nominal (or engineering) strain $\varepsilon_{\text {nom }}$ is defined as:

$$
\varepsilon_{\text {nom }}=\frac{\Delta l}{l_{0}}
$$


Where $\Delta l$ is the elongation of a gauge section with an initial length $l_{0}$. The plastic strain $\varepsilon_{p}$ can then be derived as follows:

$\varepsilon_{p}=\varepsilon_{\text {nom }}-\frac{\sigma}{E}$

Where $\sigma$ and $E$ are respectively the nominal stress and Young's modulus. Correspondingly, the true plastic strain can be estimated by substituting the nominal stress and strain with its true values. Logarithmic or true Euler-Almansi strain from DIC is given by:

$\mathcal{E}_{l n}=\ln V=\ln \sqrt{F F^{T}}$

In which $V^{2}=F F^{T}$ is the left stretch tensor and $F$, the deformation gradient. Strain components $\mathcal{E}_{i j}(i, j=1,2,3)$ can be decomposed into an elastic strain $\mathcal{E}_{i j}^{e}$ and a plastic strain $\mathcal{E}_{i j}^{p}$, that is, $\varepsilon_{i j}=\varepsilon_{i j}^{e}+\varepsilon_{i j}^{p}$. Adopting classical J2- (or Mises) plasticity, the equivalent plastic strain $\mathcal{E}_{e q}^{p}$, (or PEEQ) defined as work-conjugate of the equivalent stress, can be calculated from the deviatoric plastic strain components $e_{i j}^{p}$ :

$\varepsilon_{e q}^{p}=\int_{0}^{t} \dot{\varepsilon}_{e q} d t=\int_{0}^{t} \sqrt{\frac{2}{3} \dot{e}_{i j}^{p} \dot{e}_{i j}^{p}} d t$

In the principal strain space, based on the assumption of conservation of volume in the plastic regime, i.e., $\dot{\mathcal{E}}_{I}^{p}+\dot{\mathcal{E}}_{I I}^{p}+\dot{\mathcal{E}}_{I I I}^{p}=0$, eq. 4 reduces to:

$\varepsilon_{e q}^{p}=\int_{0}^{t} \sqrt{\frac{4}{3}\left(\left(\dot{\varepsilon}_{I}^{p}\right)^{2}+\left(\dot{\varepsilon}_{I}^{p}{\dot{\varepsilon_{I I}}}^{p}\right)+\left(\dot{\varepsilon}_{I I}^{p}\right)^{2}\right)} d t$

Where $\mathcal{E}_{I}=\ln \left(1+\frac{\partial u_{x}}{\partial X}\right)$ and $\mathcal{E}_{I I}=\ln \left(1+\frac{\partial u_{y}}{\partial Y}\right)$ are the principal logarithmic strains measured directly on the specimen surface at the necking section. $\mathrm{X}$ and $\mathrm{Y}$ are the reference coordinates along the principal axes, and $u_{x}$ and $u_{y}$ are the corresponding displacements.

The temperature evolution in the centre of the sample's gauge section is monitored using a Pt100 thermocouple with an exposed tip. The sensor used in conjunction with a PID controller 

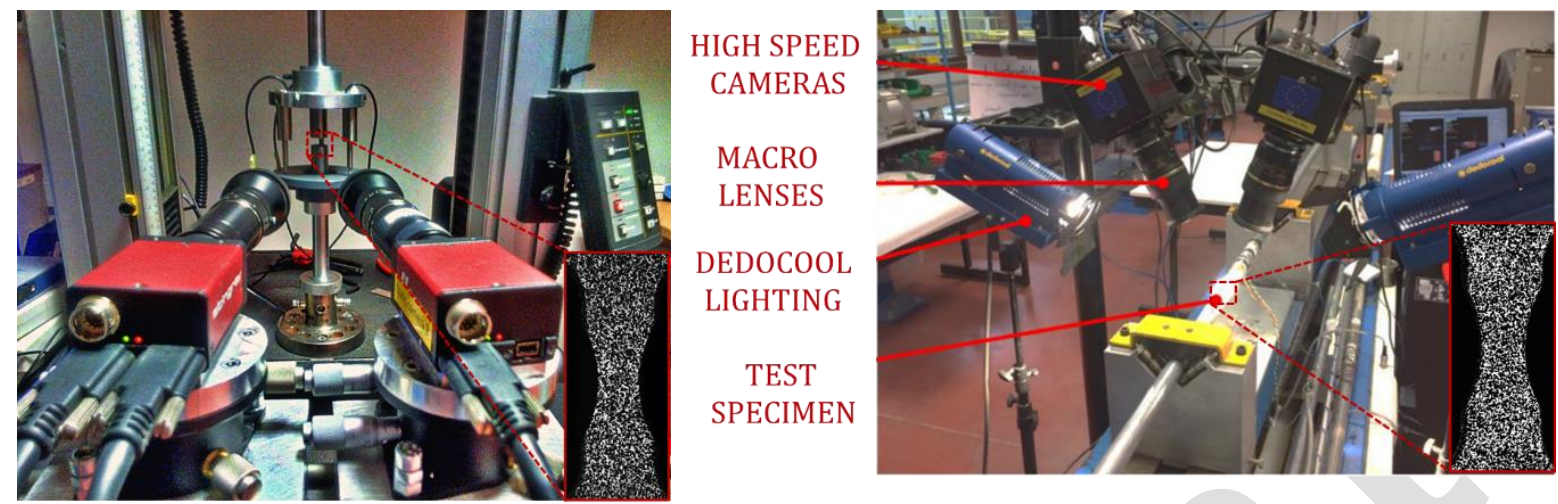

Fig 1. DyMaLab static (left) and dynamic (right) tensile test setups (Inset: Speckled notched R4.5 specimen)

$\sigma_{t r}=\sigma_{\text {nom }}\left(1+\varepsilon_{\text {nom }}\right)$

$$
\sigma_{t r}=\frac{F}{A}
$$

True stress for the notched tensile and shear samples is defined as:

$217 \quad \mathrm{~F}$ is the applied force and $\mathrm{A}$ is the instantaneous area of the specimen minimum cross-section.

218 The area of the notched samples is estimated as the product of the in-plane width reduction and 
out of plane thickness reduction calculated from the post-processing of DIC results.

Symmetrical out of plane displacement about the specimen mid-thickness plane is assumed to

221

222

229 derive the variations in the sample thickness throughout the deformation. For the shear sample, the area is defined as the product of length and thickness of the shear region considering the condition of pure shear within this zone.

From the engineering stress-strain curves, the yield strength (YS) is calculated as the offset stress at $0.2 \%$ strain as per EN ISO 6892-1:2019 [International Organization for Standardization, 2019]. The ultimate tensile strength (UTS) is the highest nominal stress reached. The fracture surfaces of the tested specimens are investigated using an FEI Quanta 450f SEM.
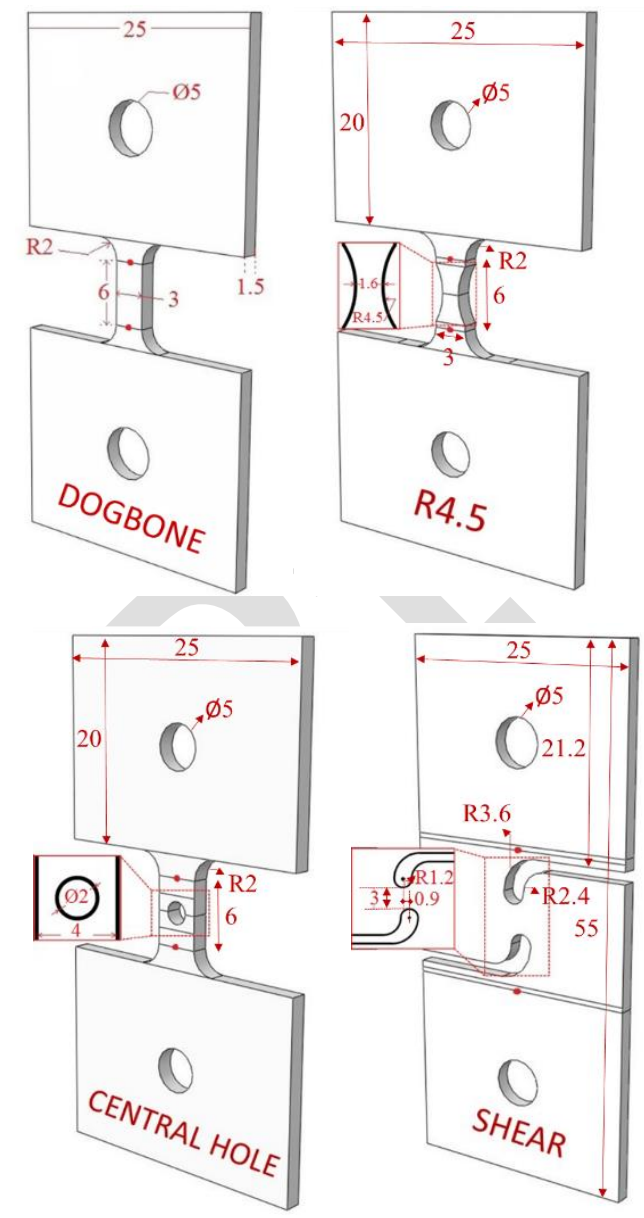
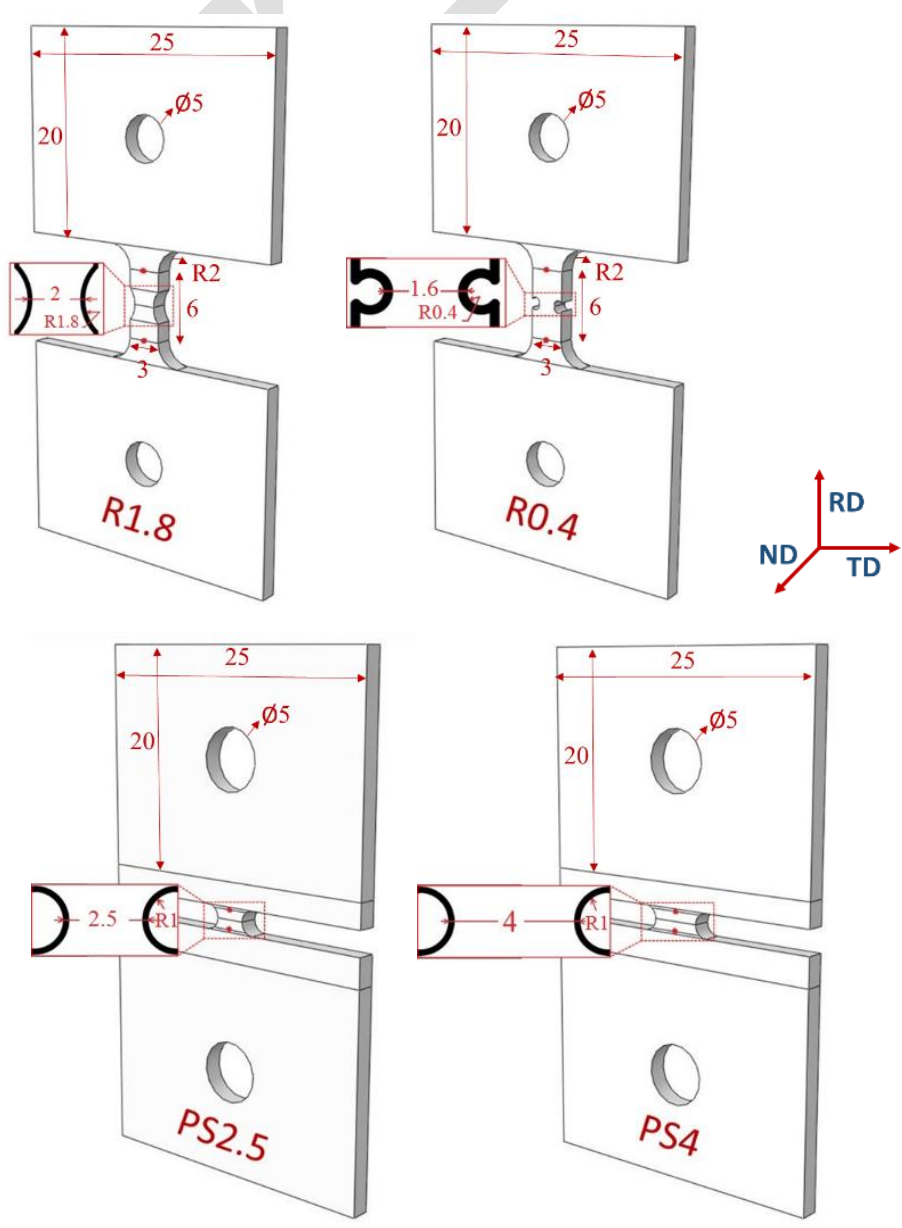

Fig 2. Purpose-designed specimens for plasticity, damage and fracture characterization of DP1000 steel at static, intermediate and high strain rates. Dimensions are given in $\mathrm{mm}$. 
234 During testing, a major fraction of plastic work is converted into heat resulting in a temperature

235 rise in the sample. The tests at the lowest speed are quasi-isothermal, however, as will be shown 236 in section 3.1, in the SHTB tests adiabatic conditions prevail. To isolate the effect of 237 temperature and unmask the true strain rate sensitivity of the material, a correction procedure 238 is introduced. To this purpose, tensile tests have been carried out at a low, isothermal strain rate 239 of $0.0001 \mathrm{~s}^{-1}$ at $-40^{\circ} \mathrm{C}$, room temperature and $100^{\circ} \mathrm{C}$, see Fig. 3a. In Fig. 3b, the ratios of the stress at a certain temperature divided by the reference, room temperature stress is represented for different values of true plastic strain before necking. These data points are used to fit a
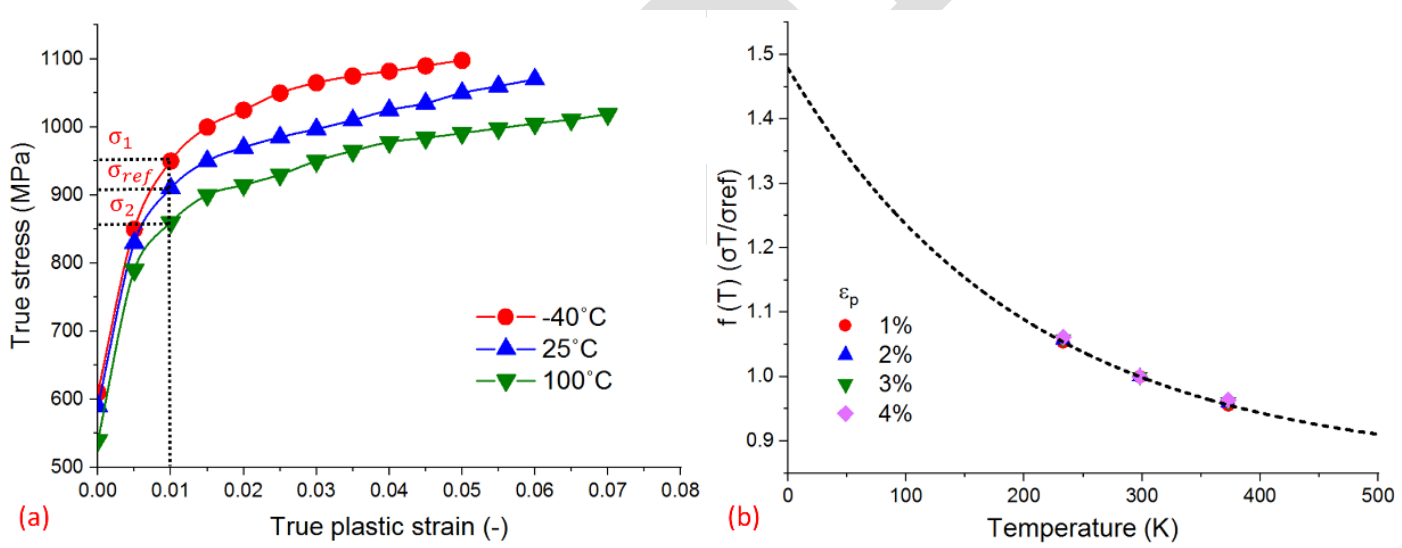

Fig 3. (a) True stress-plastic strain curves of tensile specimen at $-40,25$ and $100^{\circ} \mathrm{C}$ and (b) temperature dependence function $\mathrm{f}(\mathrm{T})$ with $c_{1}^{T}, c_{2}^{T}$ and $c_{3}^{T}$ taking the values of $0.620146,0.004954$ and 0.858314 respectively. 
253 To identify the plastic properties at different strain rates, a conventional dogbone sample is

254

255 used. Aiming at a systematic analysis of the effect of stress state on the damage and fracture properties of the DP1000 steel, 7 sample geometries are designed to be tested in conventional test setups. The geometrical optimization is performed using a finite element based parametric analysis procedure. A Levenberg-Marquardt algorithm is employed in Python programming language to minimize a cost function and, as such, to attain the solution of the optimization problem. SHBT tests require the use of small samples. Identical samples and boundary conditions are employed for static, intermediate and dynamic tests to avoid discrepancies and to ensure that that differences between the results can be fully assigned to the deformation rate. For damage studies, the stress state in a material is usually characterized by stress triaxiality $\eta$ and Lode angle parameter $\bar{\theta}$. Stress triaxiality is defined as the ratio of hydrostatic pressure $\left(\sigma_{\mathrm{m}}\right)$ and von Mises equivalent stress $(\bar{\sigma})$ :

$\eta=\frac{\sigma_{m}}{\bar{\sigma}}$

The Lode angle parameter is related to the normalized third deviatoric stress invariant and is expressed as:

$\bar{\theta}=1-\frac{2}{\pi} \cos ^{-1}\left\{\frac{3 \sqrt{3}}{2} \frac{J_{3}}{J_{2}^{3 / 2}}\right\}$

Where $J_{2}$ and $J_{3}$ are the second and third deviatoric stress invariants. A detailed description of the formulation and the physical meaning of these parameters is given in [Bai and Wierzbicki, 2008; Lian et al., 2013]. For the sample geometry optimization, it is assumed that the damage initiation, as a precursor to the failure, occurs at the location of maximum plastic equivalent strain (PEEQ). This location is denoted as the critical location and the element(s) encompassing this location is defined as the critical element(s). The main objectives of the optimization process are the following: 
1) The critical element(s) should yield the desired stress state during plastic deformation over damage initiation till fracture.

2) The critical elements should remain at the same location.

The finite element simulations are performed using Abaqus/explicit. An isotropic von Mises plasticity model is incorporated since a quasi-isotropic mechanical behaviour is reported for similar dual-phase (DP) steels [Lian, 2015; Roth and Mohr, 2018; Santos et al., 2019]. The hardening behaviour is characterized by a uniaxial tensile test under quasi-static conditions on the DP1000 flow curve at room temperature for a large strain range.

$\sigma_{t r}\left(\varepsilon_{p}\right)=\alpha \cdot A\left(\varepsilon_{0}+\varepsilon_{p}\right)^{n}+(1-\alpha) \cdot\left[k_{0}+Q\left(1-\exp \left(-\beta_{v} \varepsilon_{p}\right)\right)\right]$

$\sigma$ and $\varepsilon_{p}$ are the true stress and true plastic strain, respectively. A, $\varepsilon_{0}, \mathrm{n}, \mathrm{k}_{0}, \mathrm{Q}, \beta_{\mathrm{v}}$ and $\alpha$ are the material parameters. The first term of the equation refers to the Swift law, the second to the Voce law. $\alpha$ is a weighting parameter. The resultant flow curve is shown in Fig. 4. For all specimens, the finite element analysis is performed up to a maximum PEEQ value of around 0.6, reported as a realistic value for the fracture strain for several dual-phase steels [Lian et al., 2013; Tasan et al., 2014]. Where possible, geometrical and loading symmetries are exploited: one-eighth is modelled of the notched and unnotched tensile samples and one half of the inplane shear sample. Regular solid elements with reduced integration points (C3D8R) are applied. In the critical region, an element size of $0.1 \mathrm{~mm} \times 0.1 \mathrm{~mm} \times 0.1 \mathrm{~mm}$ is chosen for all specimen geometries since this size is similar to the length of a detectable micro-crack in the tests. Additionally, a convergence analysis shows that further reducing the mesh size does not result in significant changes in local stress and strain, as was also found in [Lian et al., 2013]. 


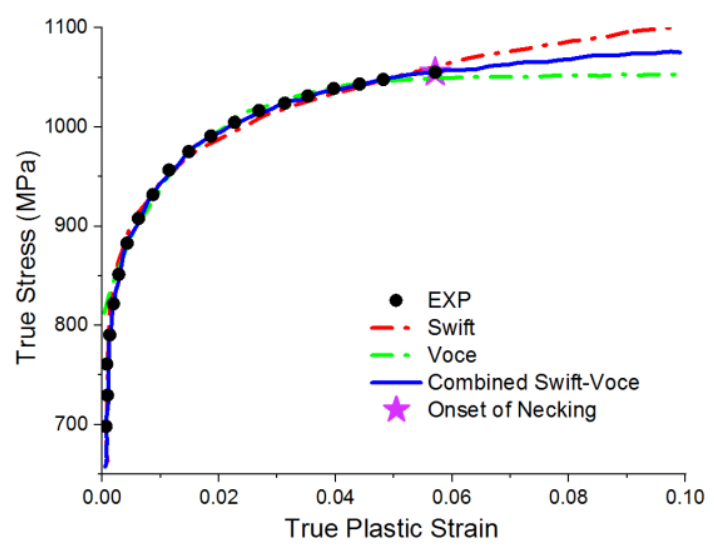

Fig 4. Experimental and extrapolated flow curve according to a combined Swift-Voce law (Eq. 12). The material constants are: $\mathrm{A}=1305 \mathrm{MPa}, \mathcal{E}_{0}=2.25 \mathrm{e}-14, \mathrm{n}=0.0768, \mathrm{k}_{0}=776.28 \mathrm{MPa}, \mathrm{Q}=265.49 \mathrm{MPa}, \beta_{\mathrm{v}}=74.34$ and $\alpha=$ 0.5 .

a) Sample geometry for identification of plasticity behaviour

The plasticity behaviour of DP1000 steel at different strain rates is characterized by uniaxial tensile tests on smooth dogbone specimens in accordance with DIN EN ISO 26203-1:2018 [International Organization for Standardization, 2018]. The dogbone specimen has a gauge length of $6 \mathrm{~mm}$ and a width of $3 \mathrm{~mm}$. The short gauge length is selected to accelerate the establishment of force equilibrium under dynamic tensile testing. Moreover, it allows to achieve the high strain rates targeted in the present study.

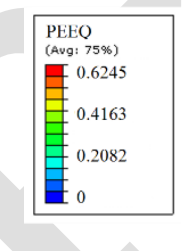

(a)
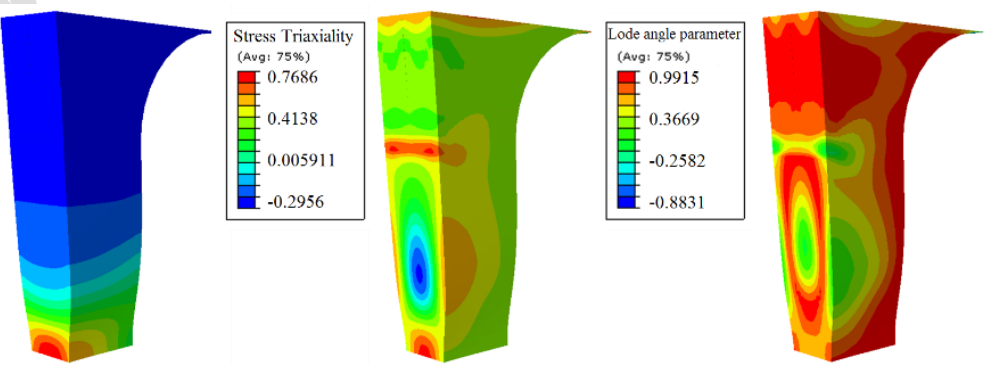

(b)
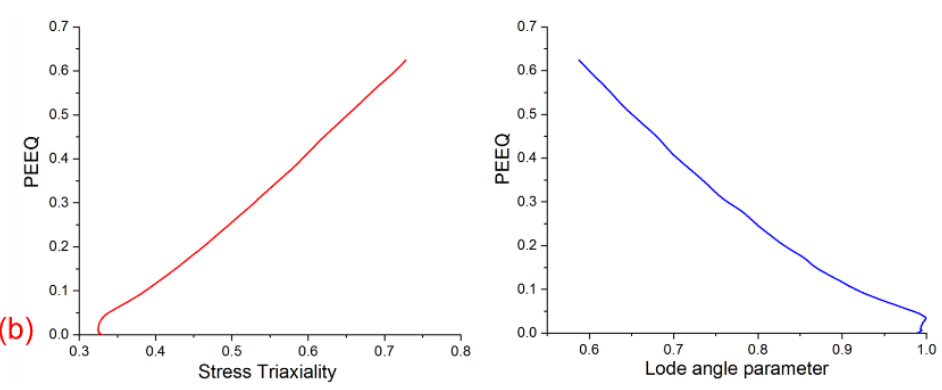
Fig 5. (a) Distribution of equivalent plastic strain, stress triaxiality and Lode angle parameter in one-fourth of the dogbone sample at a deformation resulting in a maximum equivalent strain of 0.62 and (b) loading paths in the critical element. (Best viewed in colour)

Fig. 5a displays the distribution of PEEQ, $\eta$ and $\bar{\theta}$ at a maximum equivalent plastic strain of 0.62. The loading paths, i.e., the evolution of $\eta$ and $\bar{\theta}$ with respect to PEEQ, in the critical element are shown in Fig. 5b. The critical location is concentrated around the centre of the gauge section. Because of early necking, the loading path rapidly deviates from the initial uniaxial stress state characterised by $\eta=0.33$ and $\bar{\theta}=1$, to one by $\eta=0.36$ and $\bar{\theta}=0.96$.

\section{b) Sample geometries for fracture identification under non-proportional loading}

Three notched specimens are derived from the dogbone specimen geometry presented in the previous section. As such, similar loading and measurement techniques can be adopted throughout the experimental program. The notch radius and section width at the notch are found to control the strain gradients in-plane and through the thickness of the sample and are therefore selected as the optimisation parameters. Aiming at stress triaxialities between uniaxial tension and plane strain, finally the three notched dogbone specimens, namely R4.5, R1.8 and R0.4, presented in Fig. 2 are obtained. For the three specimens, during the entire plastic deformation, the maximum PEEQ and stress triaxiality are obtained in a limited region around the centre of the gauge section, see Fig. 6 a-c. As such, the optimization target concerning the critical location is satisfied. The loading histories in the critical central element of the notched R4.5, R1.8 and R0.4 specimens, represented in Fig. 6d show that both $\eta$ and $\bar{\theta}$ diverge continuously from their initial values at the onset of yielding to values beyond plane strain.

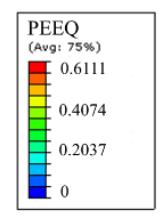

(a)

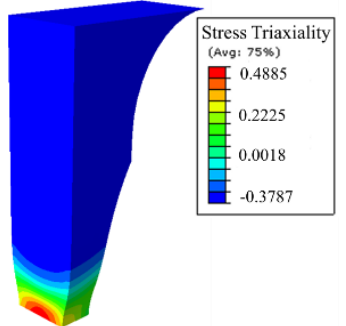

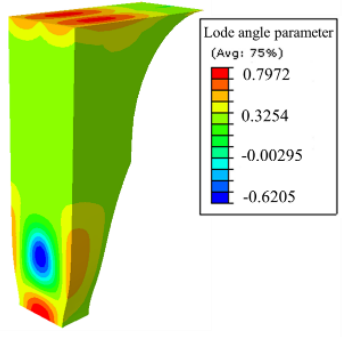

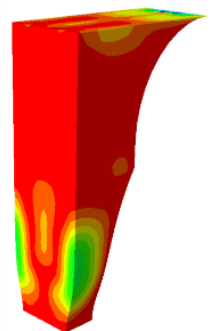




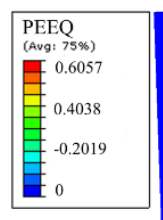

(b)
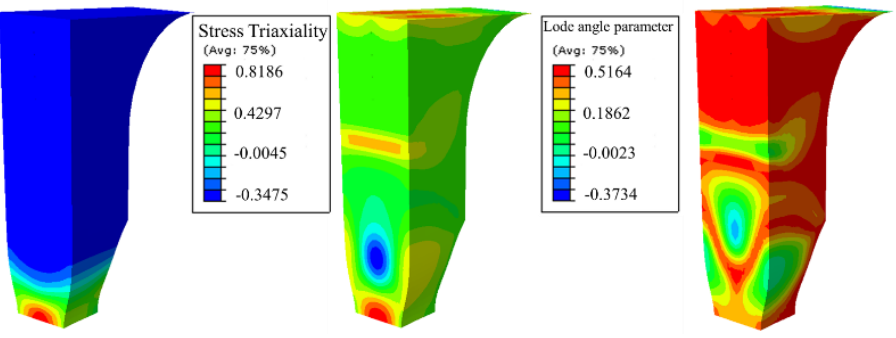

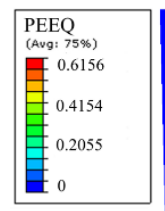

(c)
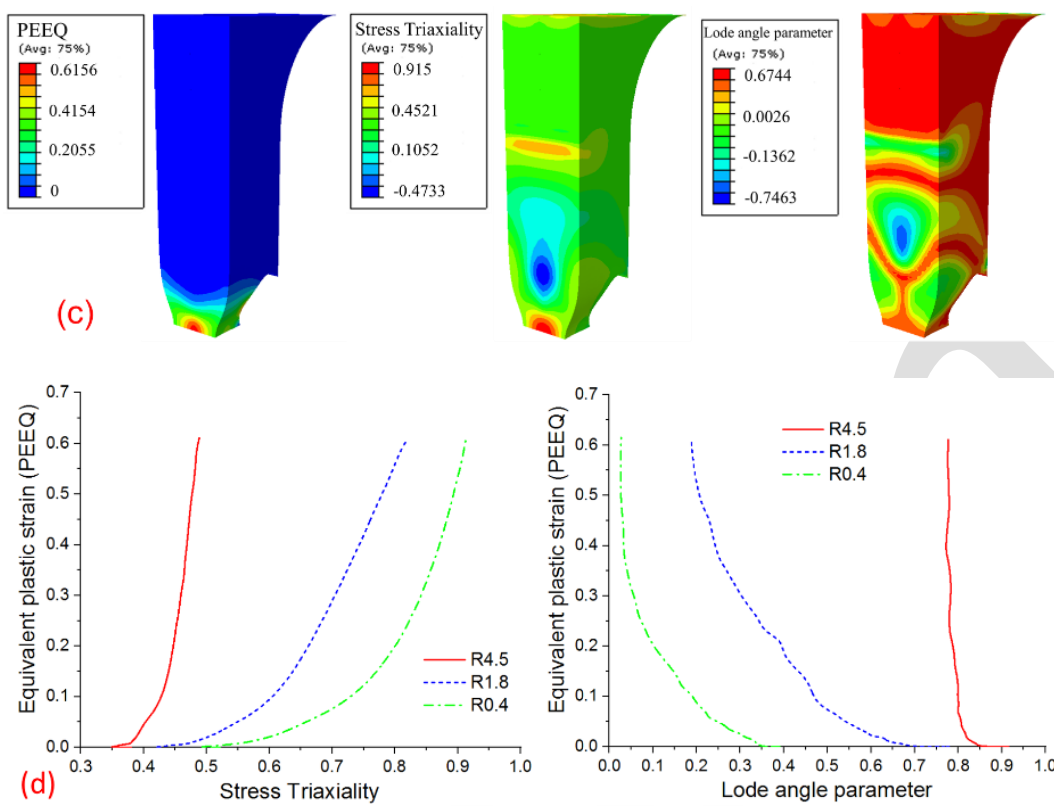

Fig 6. Distribution of the equivalent plastic strain, stress triaxiality and Lode angle parameter in (a) one-fourth of the notched R4.5, (b) R1.8, and (c) R0.4 samples at a PEEQ in the central critical element of approximately 0.6 . Loading paths at the critical element (d) of the R4.5, R1.8 and R0.4 geometries. (Best viewed in colour)

341 The geometries presented in the previous section give stress states corresponding to those in mechanisms in the DP1000 steel and to determine reliable damage and fracture loci, stable stress states are highly preferred. Therefore, four proportional specimen geometries are designed to cover a wide range of stress states: a central hole, two plane strain and an in-plane shear specimen. 


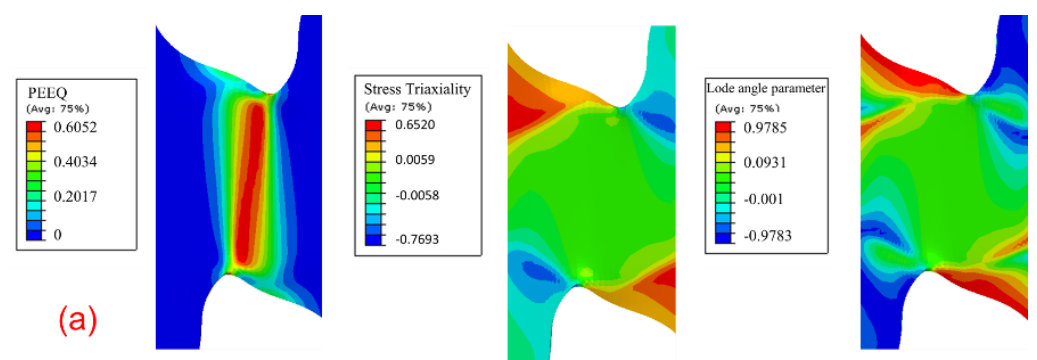

(b)
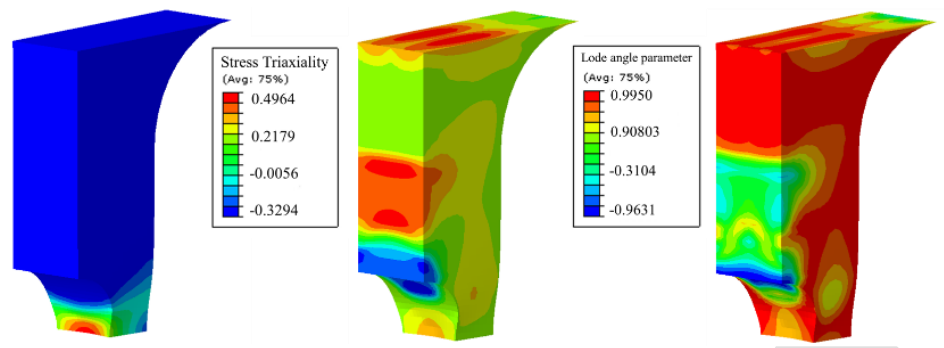

(c)
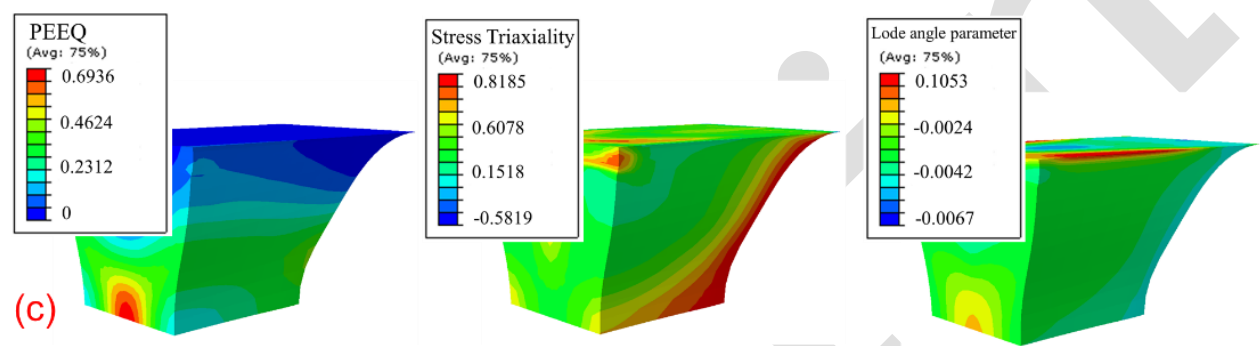

(d)
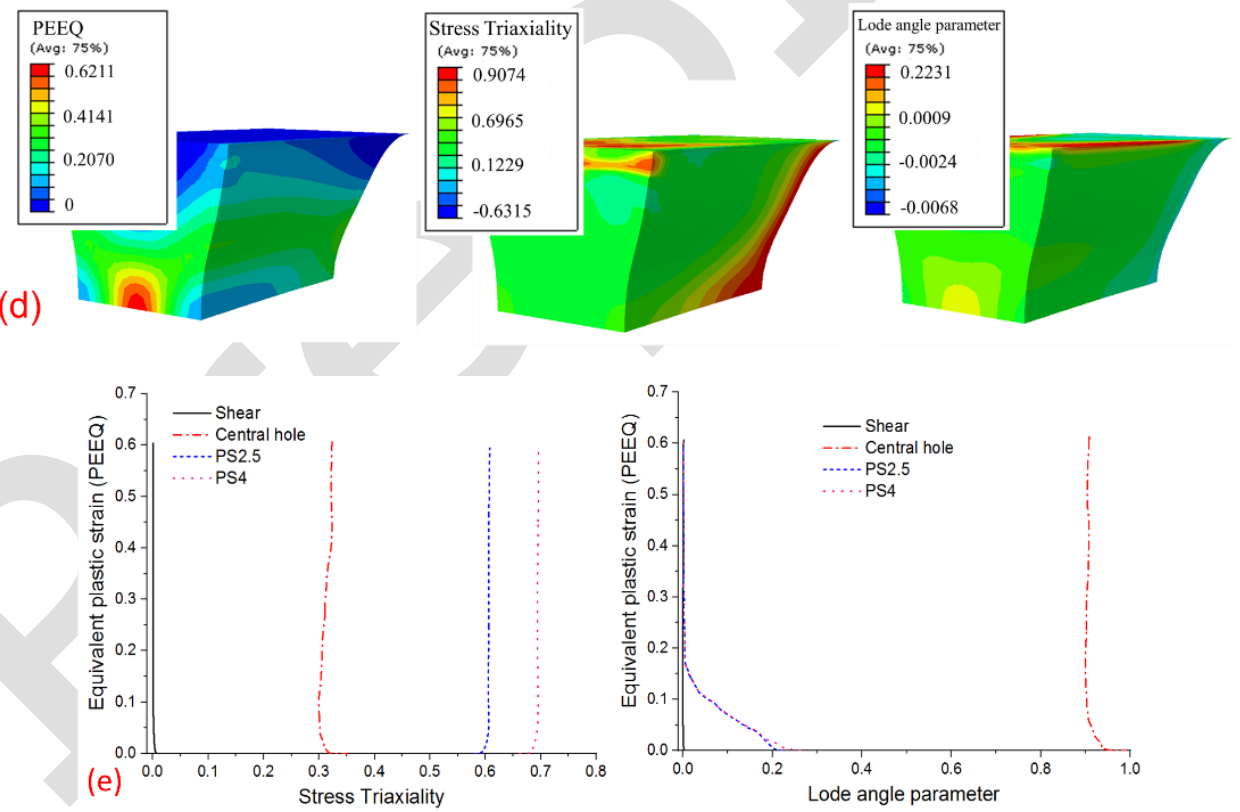

352 Fig 7. Distribution of the equivalent plastic strain, stress triaxiality and Lode angle parameter in the (a) shear, (b)

353 one-fourth of the central hole, (c) PS2.5 and (d) PS4 samples at a PEEQ of approximately 0.6 in the central critical

354 element. (e) Loading paths at the critical element of the shear, central hole, PS2.5 and PS4 geometries. (Best viewed in colour) 
For the central hole specimen, hole diameter and ligament width are the critical parameters controlling the stress state. The parametric analysis shows that larger hole sizes tend to shift the critical zone from the hole edge to the centre of the ligament, whereas smaller holes introduce larger mechanical gradients. The ligament width additionally controls the through-thickness gradients. The optimization process results in a hole diameter of $2 \mathrm{~mm}$ and a ligament width of $1 \mathrm{~mm}$. For these dimensions, the critical zone is stable and located in the middle over the thickness at the hole, see Fig. 7b. During stable plastic deformation, the stress state in the critical element features values of the stress triaxiality between 0.32 and 0.33 and a Lode angle of approx. 0.91, see Fig. 7e. Thus, values close to the values for uniaxial tension $(\eta=0.33$ and $\bar{\theta}$ 1) are obtained and maintained throughout loading.

Two plane strain samples are added to the matrix, namely PS2.5 and PS4, to feature a generalized plane strain tension state under high stress triaxialities. Both samples have the same notch radius, namely $1 \mathrm{~mm}$, whereas the widths are modified to achieve different $\eta$ values. The PS2.5 specimen, as shown in Fig. 7c and e, results in a proportional stress state $(\eta \sim 0.6$ and $\bar{\theta}$ 0.0004) in the centre of the gauge section during plastic deformation. A stable loading path is also established in the centre of the PS4 specimen, see Fig. 7d and e, however, under higher stress triaxiality, i.e., $\eta \sim 0.69$.

The shear specimen is optimized to achieve a pure shear stress state characterized by $\eta=0$ and $\bar{\theta}=0$ starting from the shear specimen geometry proposed in [Peirs et al., 2012]. The key attributes of the geometry are the length of the shear zone, the radius of the notches which delimit the shear region and the eccentricity of the notches. After optimization, a proportional shear stress state with $\eta \sim 0.0008$ and $\bar{\theta}=0.0002$ is obtained in the critical shear deformation region in and around the centre, see Fig. 7a and e, throughout the plastic deformation. Moreover, a homogenous strain distribution is observed along the shear zone with negligible thinning of 
the shear region. Experimental determination of the true material response up to large strains is thus possible.

\section{Results and Analyses}

In section 3.1 and 3.2, the results are presented of the test campaign using the samples shown in Fig. 2. In sections 3.3 and 3.4, relevant data extracted from the test series are gathered and analysed focussing on the influence of strain rate and stress state.

\subsection{Uniaxial tensile tests}

Tensile tests are carried out on the dogbone specimens at strain rates ranging from $0.0001 \mathrm{~s}^{-1}$ to $1080 \mathrm{~s}^{-1}$ averaged over the gauge length during the plastic deformation. Quasi-static (0.0001$\left.0.01 \mathrm{~s}^{-1}\right)$, intermediate $\left(1.05 \mathrm{~s}^{-1}\right)$ and dynamic $\left(350-1080 \mathrm{~s}^{-1}\right)$ loading regimes are covered. Fig. 8a presents representative engineering stress-strain curves. The DP1000 steel exhibits continuous yielding behaviour and pronounced strain hardening at all strain rates.
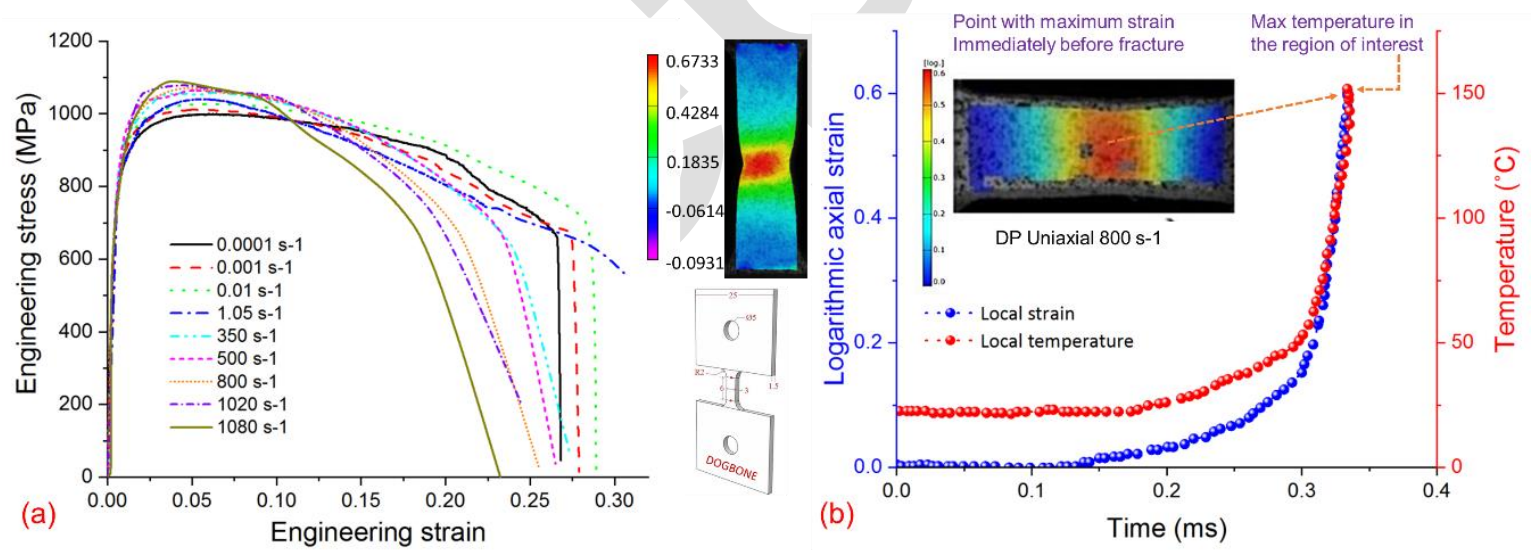

Fig 8. (a) Engineering stress-strain curves of uniaxial tensile specimens tested at quasi-static, intermediate and dynamic strain rates and (b) time evolution of temperature and plastic strain in the centre of a specimen tested at $800 \mathrm{~s}^{-1}$.

Temperature measurements performed in the centre of the samples show that isothermal conditions only exist at the lowest strain rate of $0.0001 \mathrm{~s}^{-1}$. When the test speed is increased, a measurable, though low for the static tests, the temperature increase is observed. At $800 \mathrm{~s}^{-1}$, a 


$$
\beta_{f i t}=\frac{A_{1}-A_{2}}{1+\exp \left(\frac{\left(\log \dot{\varepsilon}-x_{0}\right)}{\Delta}\right)}+A_{2}
$$

415 Where $A_{1}=-1.87442 \times 10-{ }^{16}, A_{2}=0.88, x_{0}=-1$ and $\Delta=0.66667$. From a strain rate of approximately $10 \mathrm{~s}^{-1}, \beta$ is found to saturate around a value of 0.88 , i.e., from that strain rate on adiabatic

temperature as high as $150^{\circ} \mathrm{C}$ just before failure, i.e., at a logarithmic strain of $\sim 0.6$, is captured, see Fig. 8 b. Using the temperature correction procedure described in section 2.2 .2 , the isothermal stress-strain curves at strain rates of 0.01 and $500 \mathrm{~s}^{-1}$, together with the reference curve at $0.0001 \mathrm{~s}^{-1}$, are presented in Fig. 9a. It is clear that sample heating induces significant stress softening which becomes more pronounced at higher strain rates.

Under adiabatic conditions, the temperature increase in the sample as a function of the strain is given by [Taylor and Quinney, 1934].

$\Delta T=\frac{\beta}{\rho \cdot C_{p}} \int_{0}^{\mathcal{E}} \sigma_{t r} \cdot d \mathcal{E}_{p}$

Where $\rho$ is the density of the material $(8050 \mathrm{~kg} / \mathrm{m} 3), \mathrm{C}_{\mathrm{p}}$ the thermal capacity $(466 \mathrm{~J} / \mathrm{kg} / \mathrm{k})$ and $\beta$ the fraction of mechanical energy transformed into heat or Taylor-Quinney coefficient. At lower, non-adiabatic strain rates, heat is partially dissipated to the environment resulting in a lower temperature rise than the one obtained by Eq. 13. In Fig. 9b, the $\beta$-values at necking calculated from Eq. 13 are presented as a function of the logarithm of the strain rate. The following Boltzmann-type equation is fitted to the experimental data to determine the transition curve:

$$
\text { conditions apply. }
$$



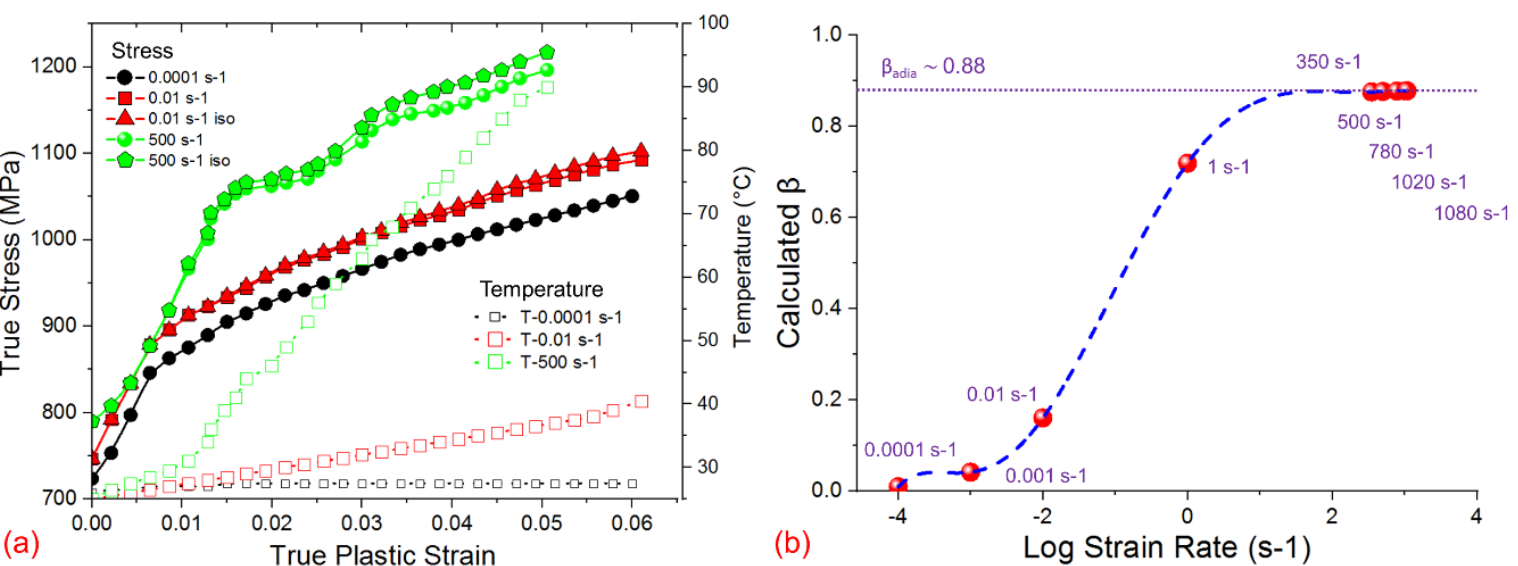

419 Fig 9. (a) Measured and corrected isothermal stress-strain curves for DP1000 steel at strain rates of 0.01 and 500

$420 \mathrm{~s}^{-1}$ together with the reference curve at $0.0001 \mathrm{~s}^{-1}$, and the temperature evolution in the centre of the sample, (b) $\beta$ -

421 value as a function of the logarithm of the strain rate together with the transition curve given by Eq. 14.

422 3.2. Fracture tensile tests

423 Figs. 10 a-g give representative engineering stress-displacement curves for the tensile tests on

424 the purpose-designed fracture specimens performed at seven different deformation speeds.

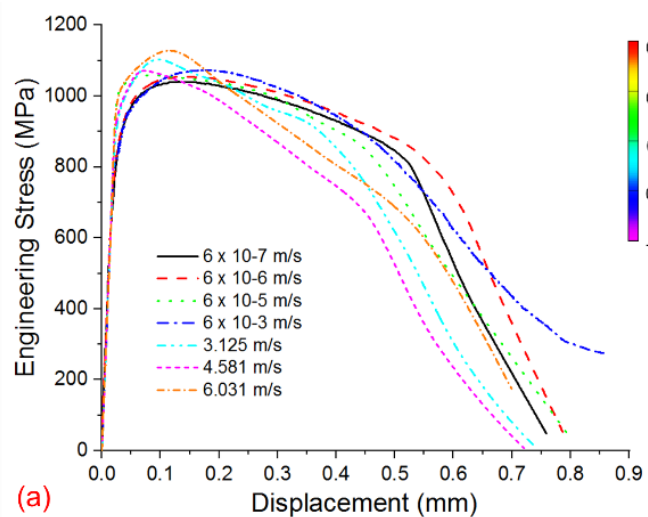

(a)

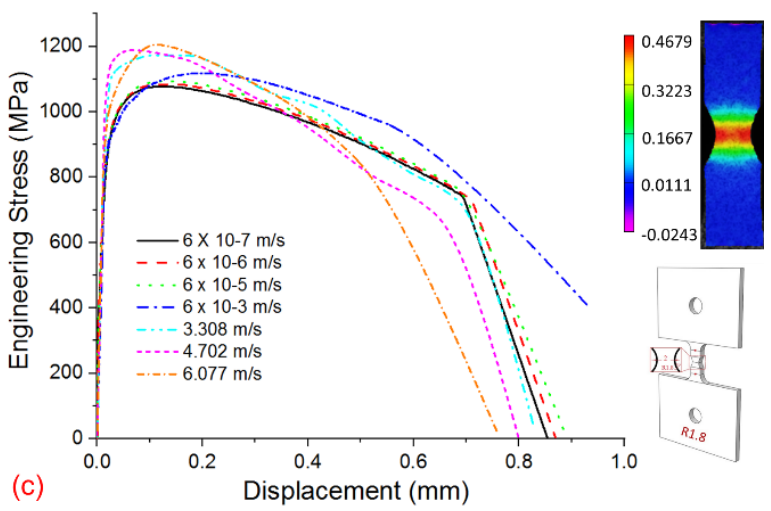

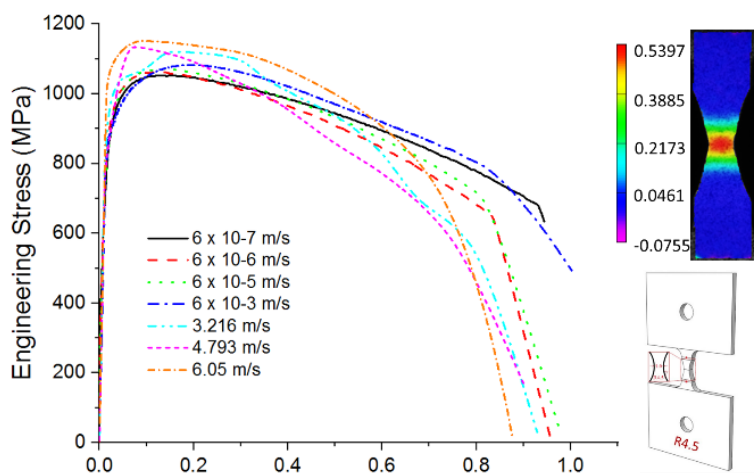

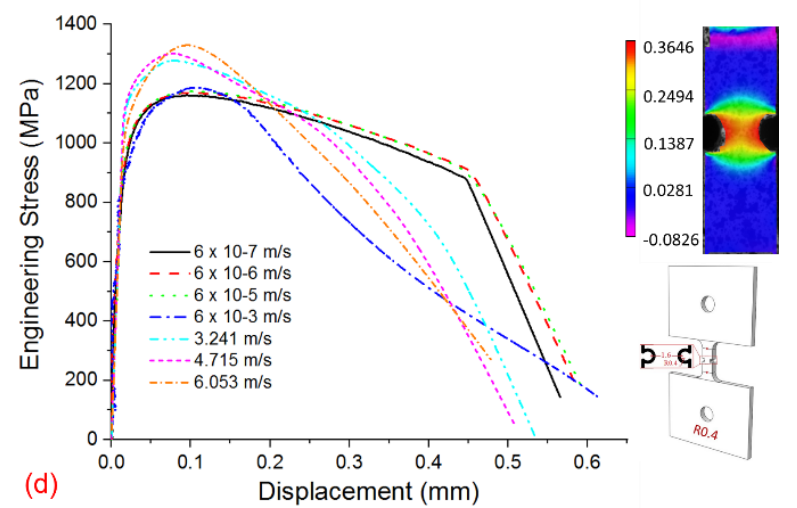

(b)

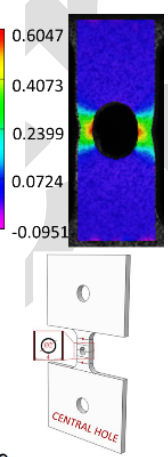

(d)

Displacement $(\mathrm{mm})$ 

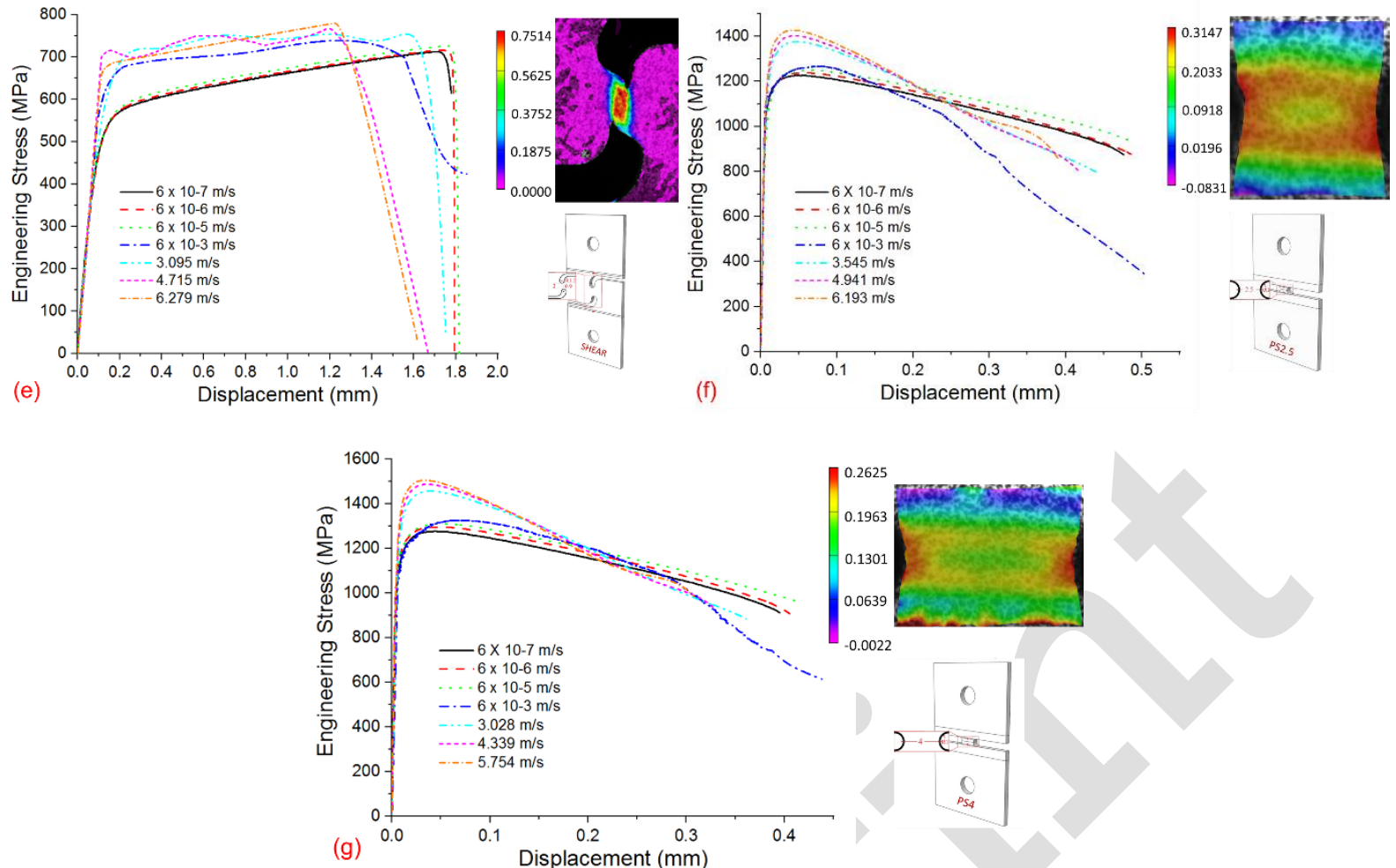

Fig 10. Engineering stress-displacement plots for tests on a) central hole, b) notched R4.5, c) notched R1.8, d) notched R0.4, e) shear, f) PS2.5 and g) PS4 samples at 7 test velocities. The velocity mentioned in the legend refers to the average relative velocity measured across the red dots using DIC. The inserted contour plot represents the logarithmic axial strain distribution before fracture for all samples except the shear sample for which the logarithmic shear strain is given.

\subsubsection{Central hole tension}

The stress-displacement curves feature a stress maximum before reaching fracture, see Fig. 10a.

Continuous yielding and significant strain hardening are observed at all the loading speeds. A positive effect of the strain rate on strength levels is clearly observed, even though thermal softening effects at the higher strain rates mask the full effect of strain rate. The logarithmic axial surface strain field plot obtained before fracture, see insert in Fig. 10a, indicates that strain localization takes place at the intersection of the hole edge and the transverse mid-plane of the specimen. Moreover, at each side of the hole, a pair of inclined localization bands is generated when the hole is stretched. Thus, strong strain gradients develop in the ligaments along the transverse direction and through the sample thickness beyond the point of local instability. 
444 Fracture initiates at the hole and propagates towards the outer edge until final rupture.

445 Increasing strain rates initially improve the displacement at failure, however, at dynamic strain

446 rates the fracture displacement decreases. Additionally, in the post-necking regime, a more

447 gradual decrease in the stress level is observed in the static tests as compared to the intermediate

448 and dynamic tests. This is partly attributed to a more localised strain distribution at higher strain

449 rates and thermal softening.

450

451

452

453

\subsubsection{Notched dogbone tension}

The engineering stress-displacement curves of the three notched samples, i.e., R4.5, R1.8 and R0.4, are presented in Fig. 10b, c and d respectively. Initial yield and maximum values of the stress increase as a function of deformation speed. Axial surface strain fields of all the notched specimens clearly indicate strong strain localisation within a narrow zone around the central cross-section area after maximum force. Thermal softening becomes prominent in this localized deformation zone, especially at the higher strain rates. This effect coupled with damage contributes to a more rapid decline in stress in the post-necking region at dynamic strain rates. Increasing deformation speeds, however, result in a non-monotonic response on the displacement at maximum force and the final displacement. For all the notched samples, these displacements increase with the strain rate in the static and intermediate zone. At dynamic rates, a descent in the displacement values is observed, even to values below the reference. The logarithmic surface strain fields inserted in Figs. 10, show that axial strain localization shifts from the centre (Fig. 10b) to the outer edge (Fig. 10d) of the specimen with increase in stress triaxiality. This may seem contradictory to the optimisation criterion that imposed a maximum value of the plastic equivalent strain in the centre of the samples. However, it should be noted that PEEQ values in the centre differ significantly from axial strain values, especially at the sample surface. In all the notched samples, fracture initiates in the centre. In the R4.5 sample, the crack propagates from the centre along the transverse axis. However, for the R1.8 and R0.4 
samples, the centre crack is rapidly followed by cracks originating at the outer edge, see section 3.5 .

\subsubsection{Plane strain tension}

Representative stress-displacement curves of the two plane strain specimens are given in Fig. 10f and g. The plane strain experiments show a significant strain rate sensitivity: a gradual increase in yield and ultimate stress is observed in the static and intermediate range. However, although the stress is not yet corrected for thermal softening, a sharp increase is obtained going from intermediate to dynamic rates. After the maximum force is reached, in the strain localization phase, the stress decreases. Partly due to thermal softening, a more rapid decrease is observed at intermediate and dynamic rates. However, as opposed to the samples discussed in sections 3.2.1 and 3.2.2, no sudden shift to a more rapid decline towards fracture is observed, except for the intermediate range. Again, a non-monotonic trend is seen for the strain rate influence on the failure displacement: in the static and intermediate regime, the failure elongation increases with the imposed deformation speed, at higher rates it decreases. Axial strain field contours (figs.10f and g) depict clear strain gradients along the specimen width with maximum values at the edges. Here again, the final fracture initiates in the centre of the sample, i.e., where the highest value of the equivalent plastic strain is reached there. However, subsequently, multiple cracks initiate along the transverse direction with increasing plastic deformation, all of which eventually coalesce leading to failure.

\subsubsection{In-plane shear}

Representative stress-displacement shear curves are shown in Fig. 10e. In contrast to the other samples, the shear samples exhibit strain hardening up to very large deformations. A minor, though positive, effect of the strain rate on the stress levels is seen under static testing velocities. Strain rate sensitivity further increases in the intermediate and dynamic strain rates. In all tests, 
512

$\theta_{u}=\frac{\sigma_{t r}^{u i s o}-\sigma_{t r}^{y}}{\varepsilon_{e q}^{u}-\varepsilon_{e q}^{y}}$

$513 \sigma_{t r}^{y}$ and $\sigma_{t r}^{u i s o}$ are the true yield and isothermal true tensile strength; $\mathcal{E}_{e q}^{y}$ and $\mathcal{E}_{e q}^{u}$ are the

the stress continuously increases up to a sudden failure. A sharp drop in stress is visible during static tests, whereas a more progressive decline is observed for all intermediate and high strain rate shear experiments. Considering global ductility, fracture elongation experiences an appreciable gain from static to intermediate strain rate tests. High strain rate conditions again reduce the final elongation. From the shear strain fields in Fig. 10e, strain localization within a narrow band around the centre is discernible. Although in the centre the highest plastic equivalent strain is obtained, since the development of damage is affected by both plastic strain and stress state, fracture might occur in the region at the notch edges where a positive stress triaxiality is obtained.

\subsection{Overview test results}

To provide a comprehensive overview of DP1000 plasticity, damage and fracture, the following sections present key properties derived from the 175 experiments considered in the study. In section 3.3.1 the focus lies on strength properties, in section 3.3.2 deformation values are comprehensibly presented.

\subsubsection{Strength levels}

Fig. 11 presents the yield strength and isothermal ultimate tensile strength and strain hardening rate obtained for the dogbone samples, normalized to the corresponding values of the reference test, as a function of the logarithm of the strain rate. The strain hardening rate at ultimate tensile strength $\left(\theta_{u}\right)$, is calculated by:

$$
\text { equivalent strains at yield and maximum force obtained by DIC. The introduced strain }
$$
hardening rate is used to identify the general hardening response and does not fully represent 
the hardening definition devised in plasticity models. An approximate curve that follows the experimental strength values is determined using the following function:

$\sigma_{f i t}^{\text {nom }}=A_{1}+A_{2} \cdot \exp \left(\frac{\log \dot{\varepsilon}}{A_{3}}\right)+A_{4} \cdot(\log \dot{\varepsilon})^{2}$

$A_{1}=(1.044293,1.036886), A_{2}=(0.00063,0.000974), A_{3}=(0.578374,0.711387)$ and $A_{4}=(-$ $0.00292,-0.00245)$ are the fitted parameters. The first and second quantity within the brackets is related to the normalised yield and tensile strength, respectively. Both $\sigma_{t r}^{y}$ and $\sigma_{t r}^{u i s o}$ increase with strain rate, however, the increment in $\sigma_{t r}^{y}$ is more prominent than in $\sigma_{t r}^{u i s o}$. The strain hardening rates in Fig. 11 indicate a depreciating hardening capacity at higher deformation speeds. Although at the onset of yielding higher initial strain hardening rates are experienced by the material with the increase in strain rate, the strain rate hardening capacity diminishes at higher plastic deformations.

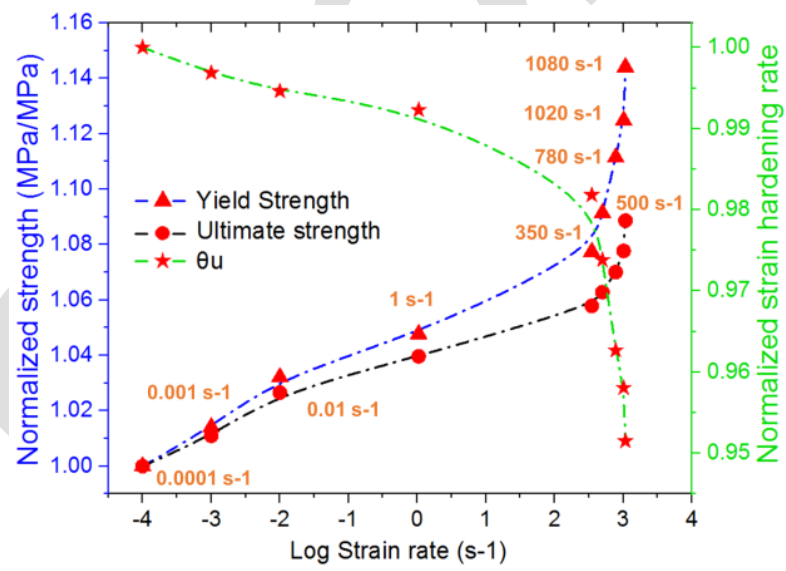

Fig 11. Evolution of normalized yield strength $\left(\sigma_{t r}^{y}\right)$, isothermal ultimate tensile strength $\left(\sigma_{t r}^{u i s o}\right)$ and strain hardening rate as a function of the logarithm of the strain rate.

Isothermal engineering stress-displacement plots for the lowest quasi-static, intermediate and middle dynamic deformation rates are presented in figs.12a, b and c, respectively. From the reference series (Fig. 12a), it is clear that the maximum stress increases considerably with stress triaxiality. However, the strength enhancement occurs at the expense of global ductility. The shear tests exhibit the lowest strength. The absence of diffuse and localized necking phenomena 

tests, on the other end, show the highest strength and lowest fracture strain.
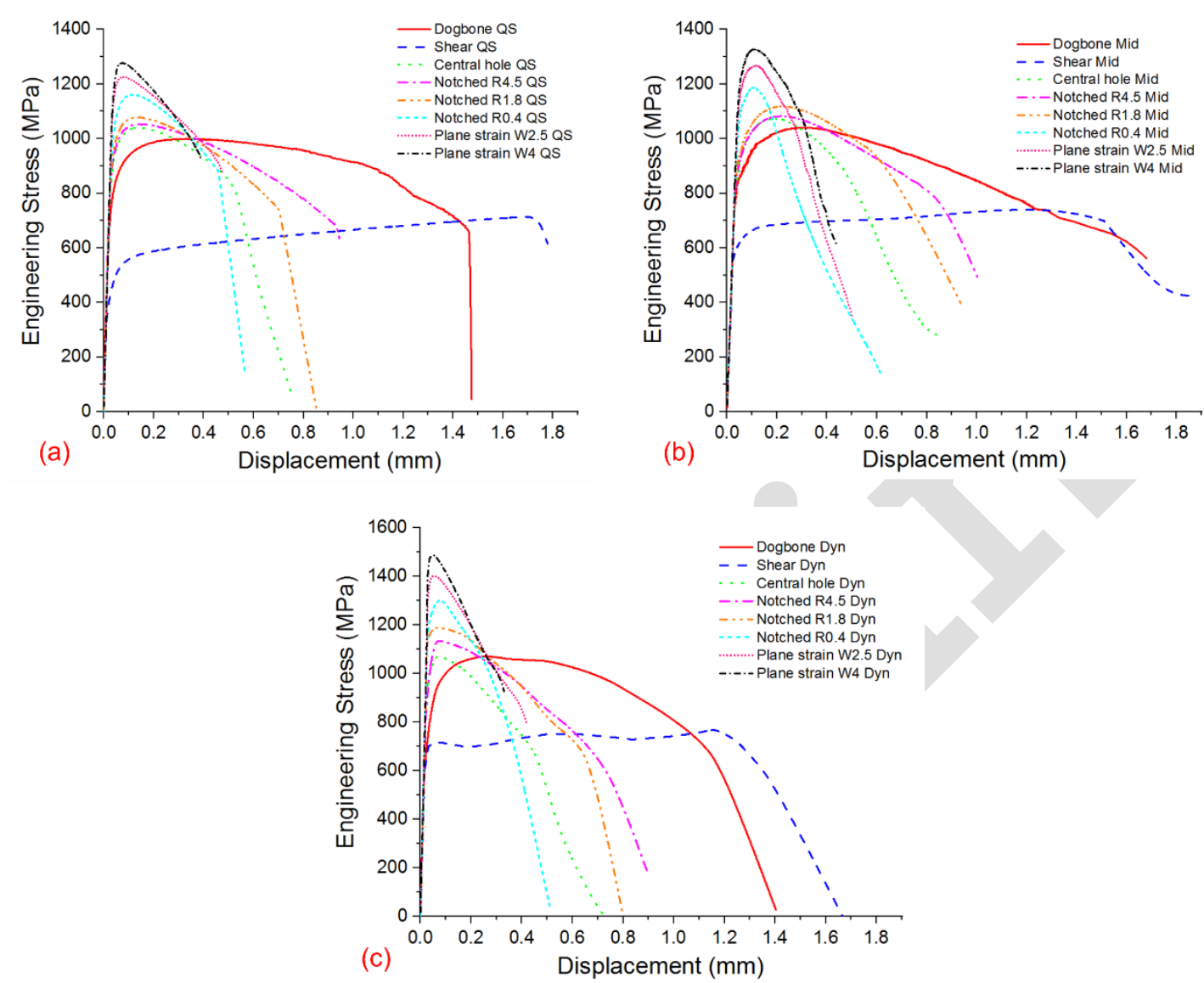

539 Fig 12. Combined engineering stress-displacement curves of damage/fracture tensile tests at different test velocity ranges: a) lowest quasi-static, b) intermediate and c) medium dynamic

541 Similar observations are made for the intermediate and high strain rate tests. However, the stress

542 drop after the severe strain localisation is less abrupt compared to the static tests.

543 To elucidate the relationship between strain rate and stress state, the evolution of $\sigma_{t r}^{y}$ and $\sigma_{t r}^{u i s o}$ 544 across the entire range of imposed stress states and strain rates is shown in Fig.13. A positive 545 strain rate sensitivity is apparent for all stress states. The largest increase in strength levels is 546 evident at the transition from intermediate to dynamic rates. The gain in strength is significantly 547 more pronounced for the samples with higher stress triaxialities. It has to be noted that the 548 deformation rate has an influence on the stress state, especially at higher levels of deformation. 

effect on the stress state at the onset of necking will be limited.

551

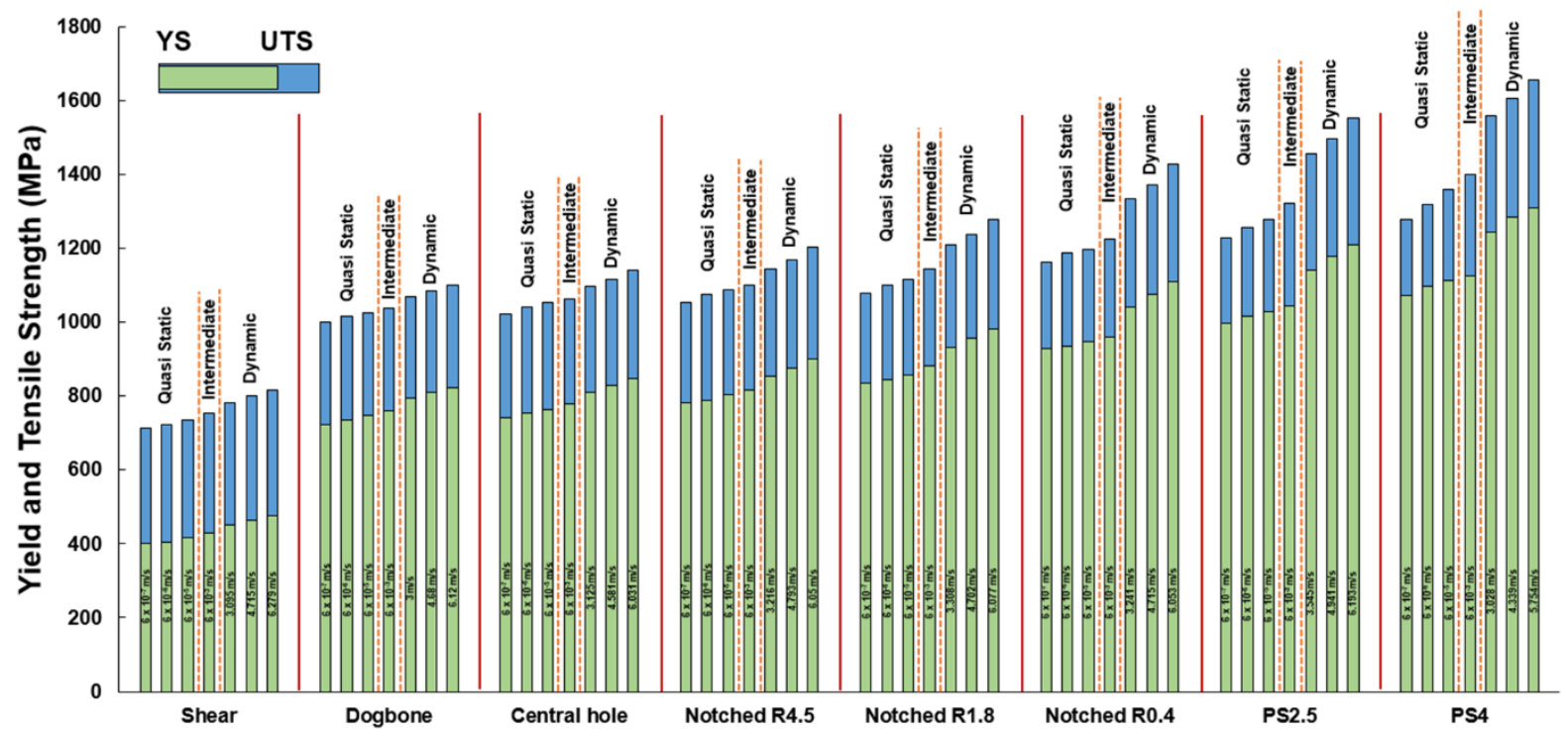

Fig 13. Effect of strain rate and stress state on the yield and tensile strength converted to isothermal values. For each stress state, the imposed deformation rate increases from left to right; the corresponding test velocities are indicated along the bars.

Values of $\theta_{u}$ at the reference deformation rate for all tested samples are given in Fig. 14a. An increase in the strain hardening rate is obtained with increasing stress triaxiality. To uncouple the influence of strain rate off the stress state, Fig. $14 \mathrm{~b}$ presents the isothermal strain hardening ability normalized to its values under quasi-static reference state, for each geometry. Strain rate clearly has a negative impact on the hardening capacity of the DP1000 steel, particularly under dynamic loading. This conclusion applies to all the tests conducted in this study. However, an increase in stress triaxiality retards and reduces the strain rate induced loss in strain hardening rate. The most pronounced degradation in hardening rate is observed for the shear stress state, the least pronounced for the highest triaxiality plane strain samples. 
564

565

566

567

568

569

570

571

572

573

574

575

576

577

578

579

580
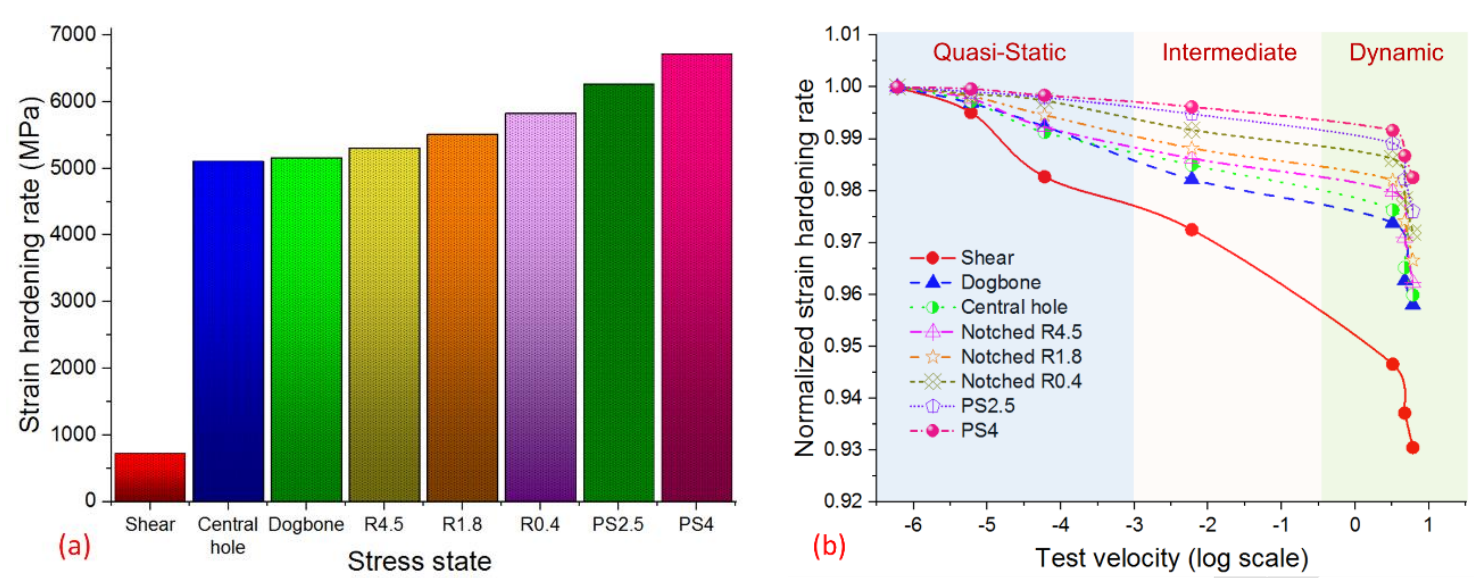

Fig 14. (a) Strain hardening rate at the lowest, reference deformation rate for all sample geometries and (b) normalized hardening rate as a function of test velocity.

\subsubsection{Uniform elongation and fracture loci}

As opposed to the strength values, increasing the strain rate results in a non-monotonic evolution of the deformation capacity of the DP1000 steel. Indeed, as can be seen in Fig. 15, which represents the uniform strain and total strain as a function of strain rate for the dogbone specimen, the elongation values increase as a function of strain rate in the static and intermediate strain rate regime, though a significant reduction is observed at dynamic strain rates. The experimental data is captured by a growth-decay function:

$\varepsilon_{f i t}=z_{0}+b \cdot \log \dot{\varepsilon}+c \cdot(\log \dot{\varepsilon})^{2}+d \cdot \exp \left(-\exp \left(\frac{\log \dot{\varepsilon}-k_{t}}{w}\right)\right)$

Where $z_{0}=(0.549568,0.779497), b=(-0.0966,-0.1111), c=(-0.00924,-0.01127), d=(-0.8014$, $-0.86843), k_{t}=(1.61302,1.193892)$ and $w=(2.38104,2.381365)$ are the estimated parameter sets. For each term, the first and the second value within the parentheses correspond to the uniform and total strain, respectively. The relatively higher enhancement in total elongation compared to uniform elongation at static and intermediate strain rates indicates that much of the gain in ductility occurs in the post-necking phase. 


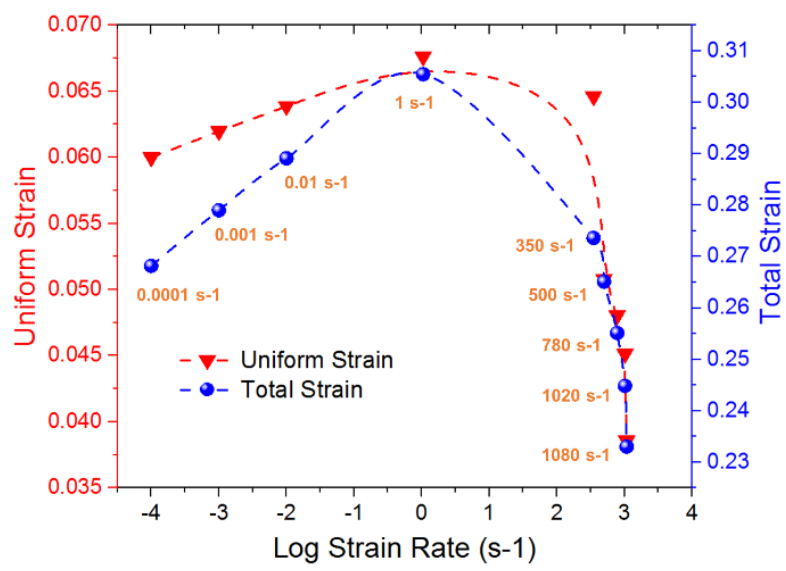

581

582

583

584

585

586

587

588

Fig 15. Evolution of uniform and total strain as a function of the logarithm of the strain rate.

Fracture elongation reflects only the global effect of strain rate and stress state on material ductility. Since in all samples the strain fields are highly heterogeneous and different volumes of the material are involved in the deformation process, local ductility values are paramount to assess the material response. To this end, DIC local equivalent plastic strains, calculated using Eq.5, are further considered. With the local ductility values, a 3D material fracture locus is established in the space of equivalent plastic strain to fracture $\left(\varepsilon_{e q}^{p f}\right)$, stress triaxiality and Lode angle parameter. To establish the relationship between material ductility and stress state for the DP1000 steel, only the proportional loading conditions are utilized. The test data are used to determine the parameters of the symmetric formulation of the $3 \mathrm{D}$ fracture locus defined in the extended modified Bai Wierzbicki model [Lian et al., 2013; Wu et al., 2017]:

$\varepsilon_{e q}^{p f}(\eta, \bar{\theta})=\left[D_{1} \cdot \exp \left(-D_{2} \eta\right)-D_{3} \cdot \exp \left(-D_{4} \eta\right)\right] \cdot \bar{\theta}^{2}+D_{3} \cdot \exp \left(-D_{4} \eta\right)$

$D_{1}, D_{2}, D_{3}$ and $D_{4}$ are the material fracture parameters. A trust region-based surface fitting optimization algorithm is employed in Matlab to determine these material parameters. For the parameter optimization, the shear, the central hole, the two plane strain and the R4.5 tensile tests are used. The R4.5 test is added to improve the quality of the fitting process. Indeed, despite the initial non-proportionality, the R4.5 specimen demonstrates a constant stress state in the later stage of plastic deformation. The thus obtained fracture locus at quasi-static strain 
rates for DP1000 steel is depicted in Fig.16. The corresponding plane stress path on the fracture locus is depicted using a red dotted line.

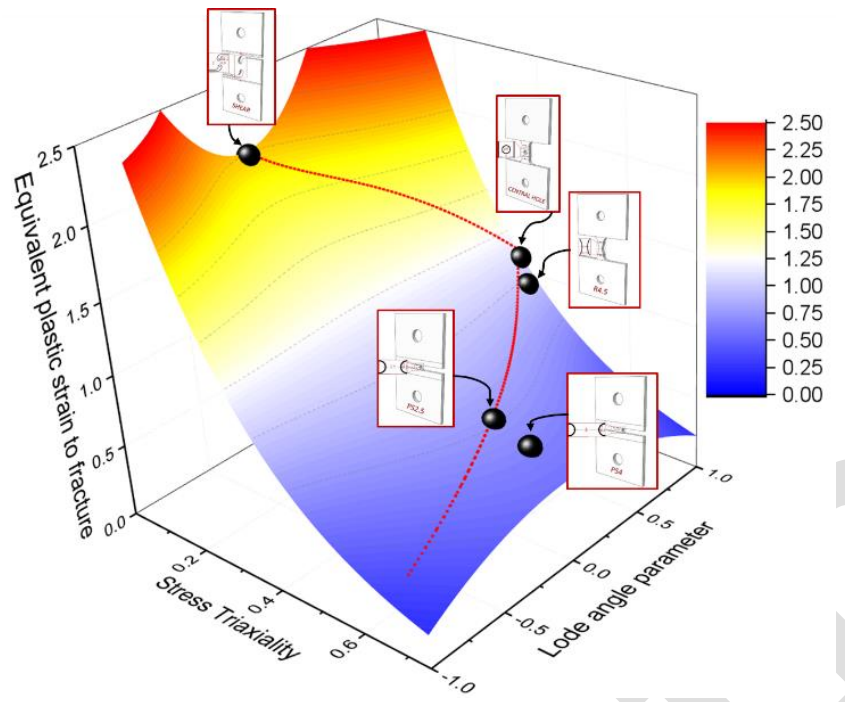

Fig 16. Fracture locus of DP1000 steel for proportional loading obtained with $D_{1}=4.11224, D_{2}=3.85212$, $\mathrm{D}_{3}=2.01749, \mathrm{D}_{4}=1.68049$. Black spheres denote the experimental data. Fracture locus for plane stress proportional loading is illustrated by red dotted lines. (Best viewed in colour)

A detrimental impact of stress triaxiality on $\varepsilon_{e q}^{p f}$ is evident. With a $\varepsilon_{e q}^{p f}$ of 1.989 , the shear sample demonstrates that fracture initiation and rupture is effectively delayed at low triaxialities. At the highest stress triaxiality, plane strain condition, on the contrary, a reduction of the $\varepsilon_{e q}^{p f}$ value of $66 \%$ is observed.

Fracture loci at intermediate and high strain rates are computed in the same way as for the static tests. Two different views of the three failure loci are presented in Fig. 17. The colour scale in the figure represents the equivalent strain level under different conditions. The ductility deterioration with increasing stress triaxiality is comparable across different strain rate intervals. However, the strain rate dependency on the fracture strain is not monotonic. Indeed, as is clear from Fig. 17, from quasi-static to intermediate strain rates, a rise in $\varepsilon_{e q}^{p f}$ is observed for all combinations of stress triaxiality and Lode angle parameter considered. A further rise in strain rate results in an adverse effect on local ductility. 

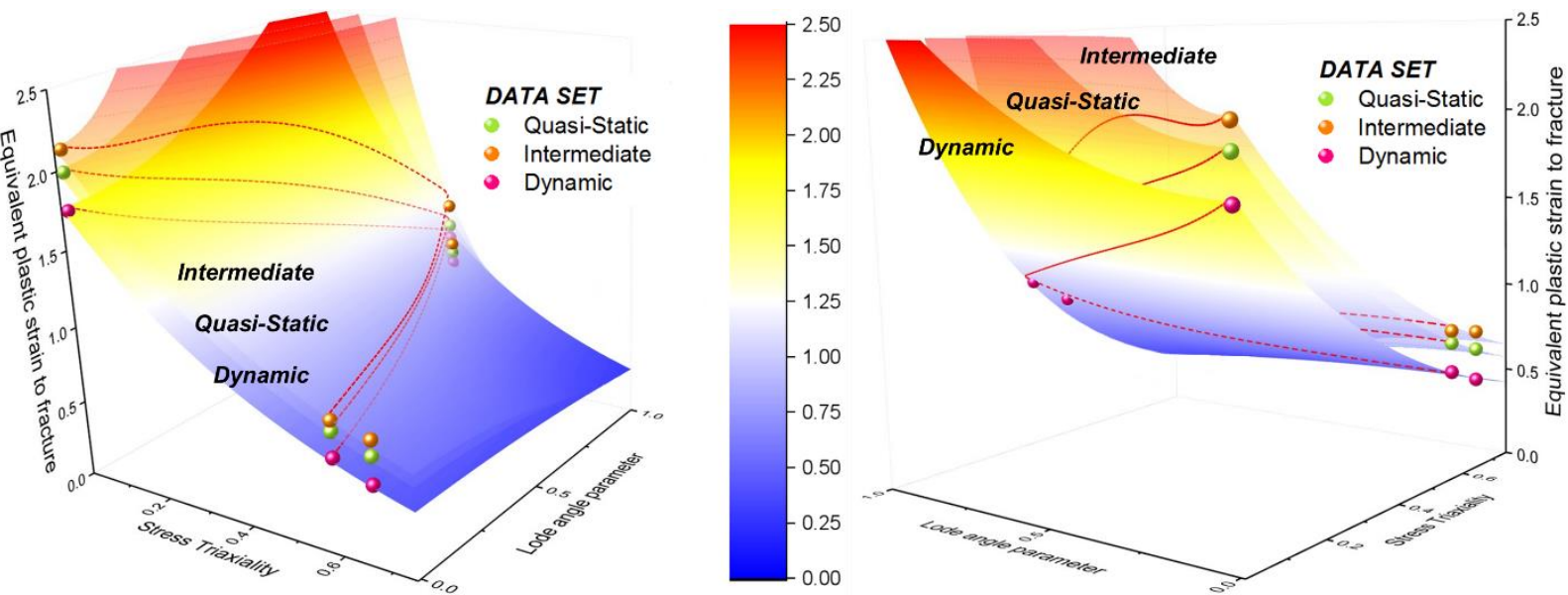

Fig 17. Failure loci (two views) at various strain rate intervals. Experimental data sets are denoted using the corresponding legends in the figure. Red dotted lines denote the plane stress proportional loading under each strain rate interval. (Best viewed in colour)

As is also the case for the hardening rate, see Fig. 14b, the most significant differences between the fracture strains in different strain rate regimes are perceived at low stress triaxialities.

The strain rate effect on $\varepsilon_{e q}^{p f}$ is visualized for different stress triaxialities in Fig.18. The figure further endorses the statement regarding the non-monotonic evolution of the fracture strain with strain rate. The following modified, non-linear, extreme value function is devised to replicate the 3D surface:

$\varepsilon_{e q}^{p f_{f i t}}=S_{1}+S_{2} \log v+S_{3}(\log v)^{2}+S_{4} \exp \left(-\exp \left\{\frac{S_{5}-\eta}{S_{6}}\right\}\right)+S_{7} \exp \left(-\exp \left\{\frac{S_{8}-\log v}{S_{9}}\right\}\right)$

The optimized function parameters are obtained using the Levenberg Marquardt iteration algorithm. $S_{1}=0.7283, S_{2}=0.01797, S_{3}=-0.0006618, S_{4}=4.05, S_{5}=-0.02496, S_{6}=-0.4968, S_{7}=$ $-3.856, S_{8}=2.514$ and $S_{9}=1.811$ are the values obtained after the regression fit. For all the considered stress states, $\mathcal{E}_{e q}^{p f}$ initially increases with loading velocity from quasi-static up to intermediate strain rates. Thereafter dynamic loading induces an earlier fracture. Furthermore, the detrimental nature of the dynamic loading on the local ductility in DP1000 steel is very evident in Fig. 18. 


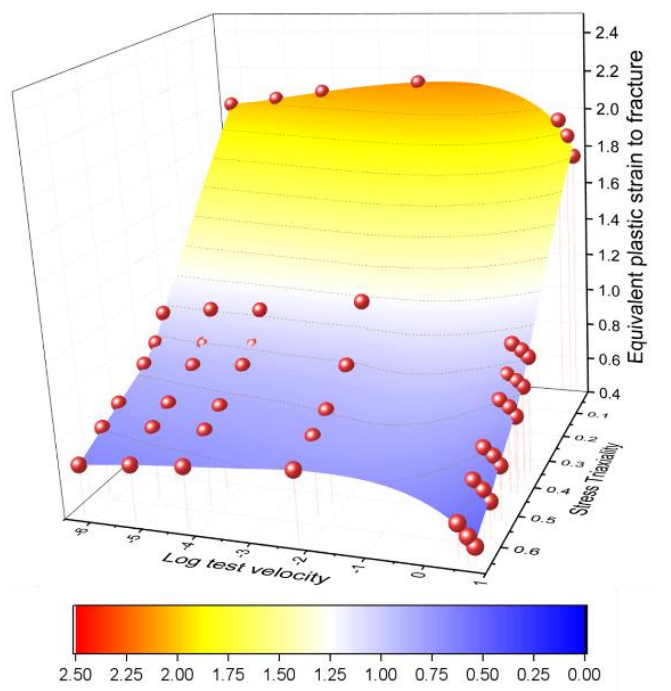

Fig 18. Effect of strain rate and stress triaxiality on equivalent plastic strain to fracture of DP1000 steel. Individual dots represent data points from experiments. (Best viewed in colour)

Equivalent plastic strains for the shear tests measured at the instant of maximum force are

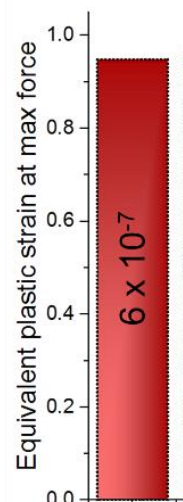

(a)
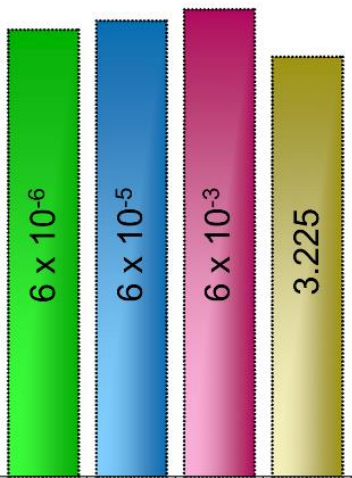

Test velocity $(\mathrm{m} / \mathrm{s})$

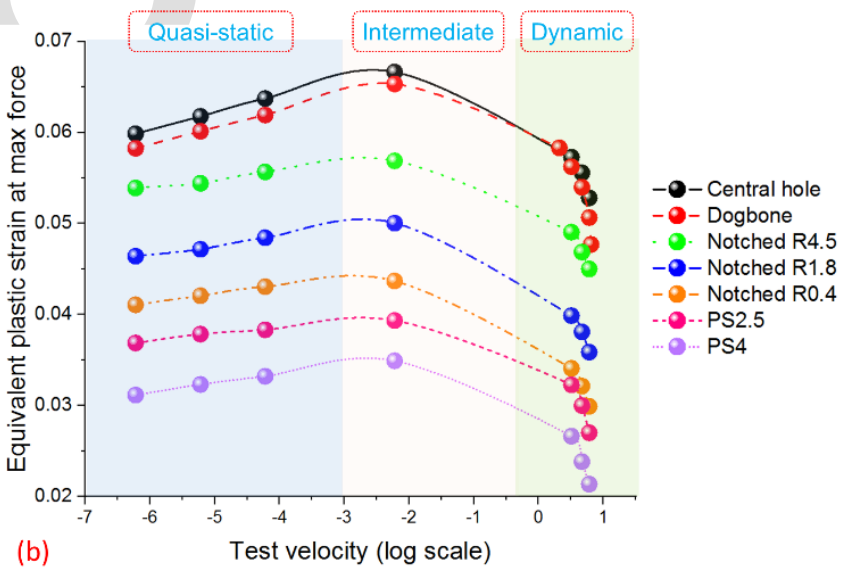

643 Fig 19. (a) Equivalent plastic strain levels for shear tests across various test velocities measured at the instant of maximum force. Corresponding test velocities are specified along with the bar plots. (b) Effect of test velocity on the equivalent plastic strain at necking for all the tensile samples

A non-monotonic evolution of the plastic strain with a rising deformation rate applies in this case as well. Here again, the equivalent plastic strain experiences an increase in its value from quasi-static to intermediate strain rates. A sharp drop is evident thereafter, upon entering the 


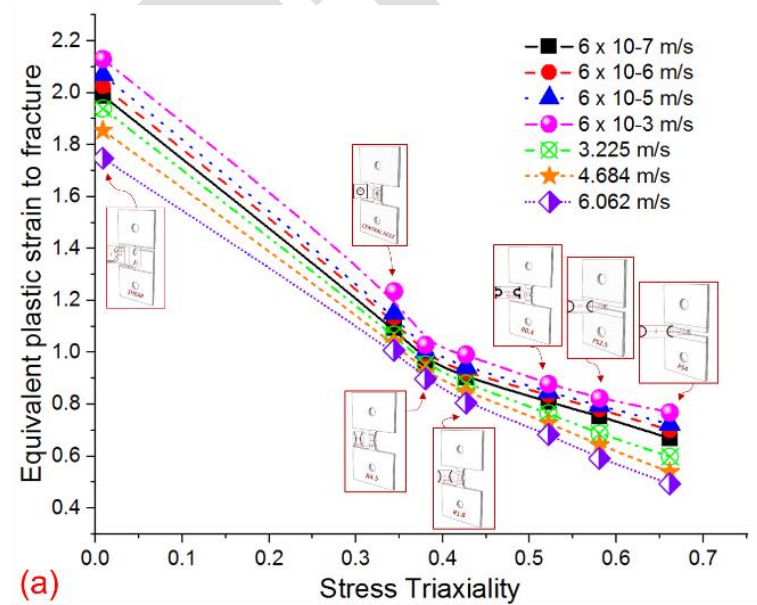
significantly to failure strain. fracture, see Fig 20a and 20b.

dynamic loading regime. It can thus be established that strain rate is highly influential from the early stage of plastic deformation until fracture for DP1000 steel. An increase in stress triaxiality limits the development of equivalent plastic strain in the material thereby triggering an earlier onset of instability. Similar to the observations in Fig 16, the shear sample exhibits the highest strain before localization/instability throughout the strain rate testing range while the plane strain sample shows the lowest. It can additionally be seen that the gain in equivalent plastic strain from quasi-static to intermediate strain rate is negatively affected by stress triaxiality, see Fig 19b. Shear samples gain the most in deformation capacity, plane strain samples the least. Loading path changes introduce a sharper drop in the strain at the onset of instability at dynamic loading rates, particularly for the R1.8 and R0.4 samples. Comparing the equivalent strain values at the onset of instability and at fracture, it is perceived that, in all the fracture tests under all the tested conditions, post instability strain localization events contribute

The evolution of $\mathcal{E}_{e q}^{p f}$ with respect to initial stress state parameters $(\eta$ and $\bar{\theta})$ is plotted across all the loading speeds to establish an overview of the impact of stress state parameters on

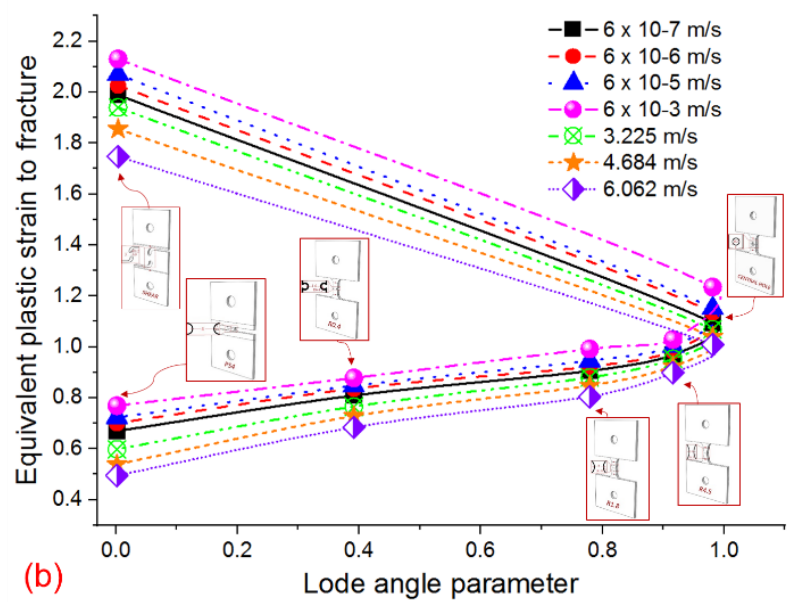

Fig 20. a) Stress triaxiality and b) Lode parameter dependencies on the fracture strain across various strain rates. 
667 Besides the dominating influence of stress triaxiality on $\mathcal{E}_{e q}^{p f}$, the effect of the Lode parameter 668 on the fracture strain becomes more pronounced with a rising deformation rate. The effect is most apparent for the shear samples, demonstrated by the highest strain rate influence on the fracture strain.

\subsection{Fractographic analysis}

672 Post mortem, the fracture surfaces of selected samples are observed by SEM. For each sample

673 series, three samples, i.e., one tested at static, one at intermediate and one at high strain rate, are selected for this analysis. Figs. $21 \mathrm{a}-\mathrm{g}$ present the fractographs at two magnifications, i.e., one showing the entire fracture surface and one with more details at the critical location, i.e., where the fracture is assumed to initiate.

677 The dependence of the $\varepsilon_{e q}^{p f}$ on the strain rate, as presented in section 3.3.2, is reflected in the 678 images of the entire fracture surfaces. Indeed, except for the shear samples, the area reduction, especially thinning, is most pronounced for the samples tested at intermediate strain rate, followed by the static and then the dynamic samples. Dimples with average sizes ranging from 1 to 5 micrometre depending on the test condition are observed on all surfaces. A process of void nucleation, growth and coalescence is clearly the primary contributor to macroscopic failure. Moreover, in the centre of the dogbone, notched and plane strain samples, and at the hole edge of the central hole sample, mainly equiaxed dimples are found typical for a tension failure which supports the assumption that fracture initiates in the critical zone. Away from the fracture initiation location, elongated dimples are observed typically for tensile tearing or shear

687 in the inclined lips formed in certain samples towards the outer sample edges.

688 Fractographs of the dogbone samples are depicted in Fig. 21a. At static and intermediate strain rates, mainly small dimples are found. However, also some larger ones with secondary dimples

690 are observed. In line with $\varepsilon_{e q}^{p f}$, the deepest dimples are seen at intermediate rates, fewer and the 
695 propagating macro crack.

696 Similar fracture features are found in the central hole samples, see Fig. 21b. Apart from a few enlarged dimples, the critical zone is mainly covered by homogenously distributed primary

698 dimples.

699
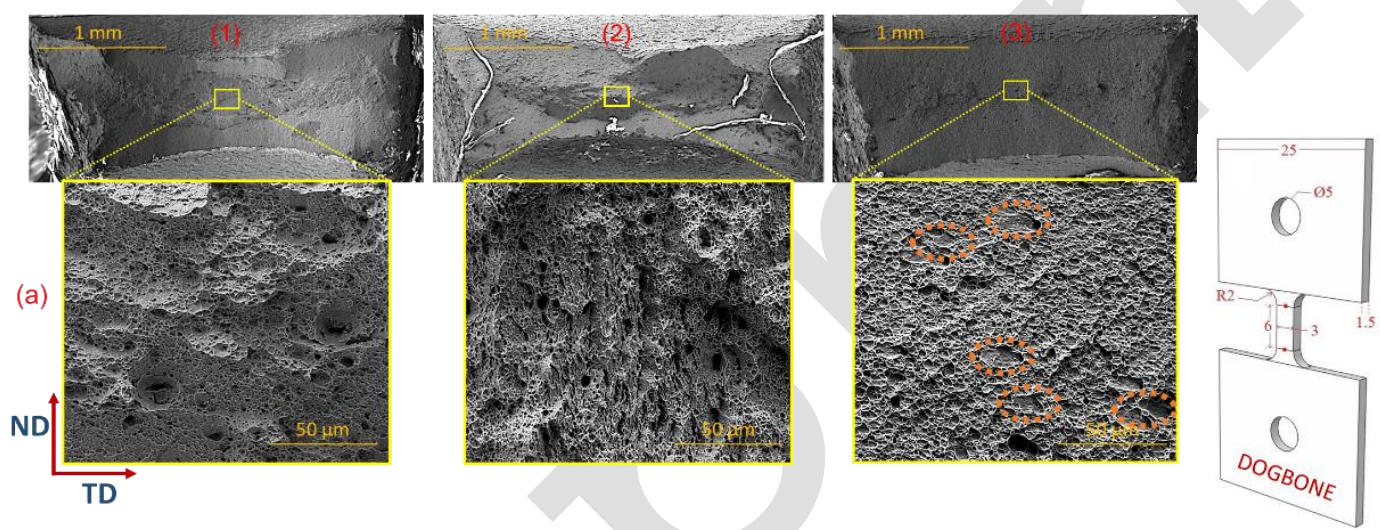

700
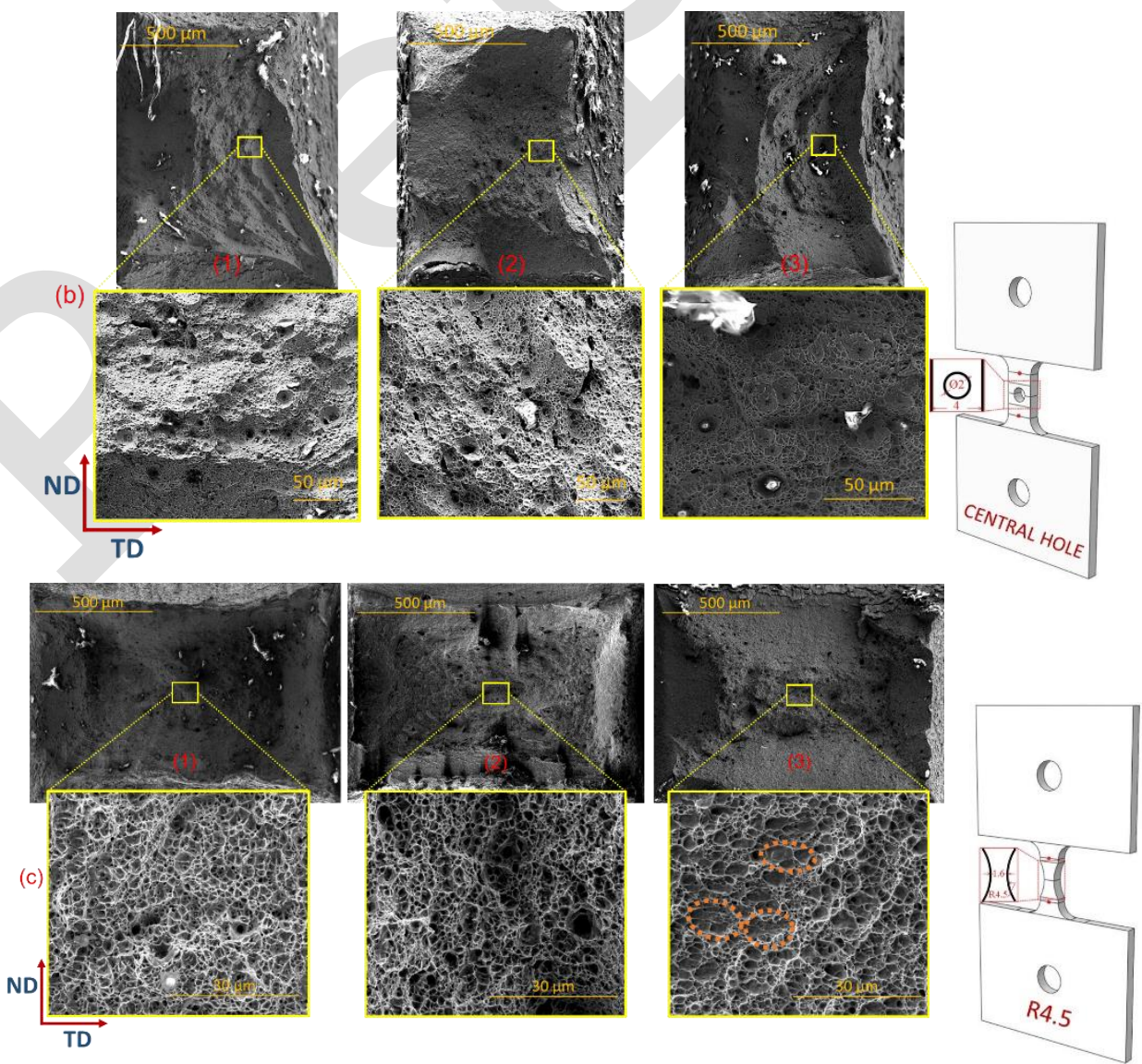

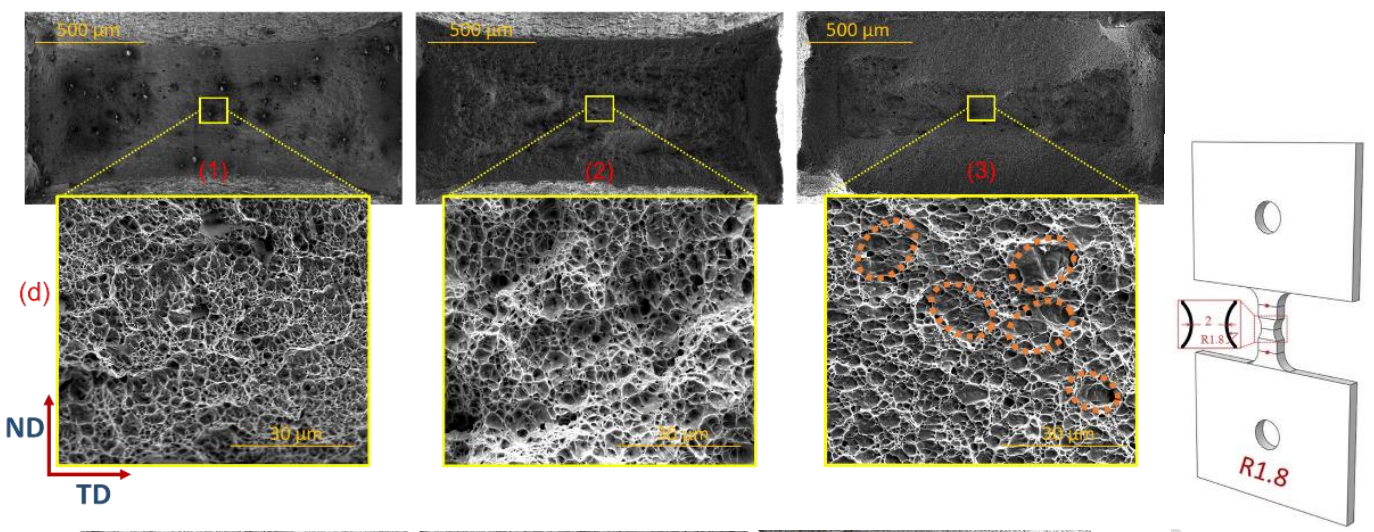

703
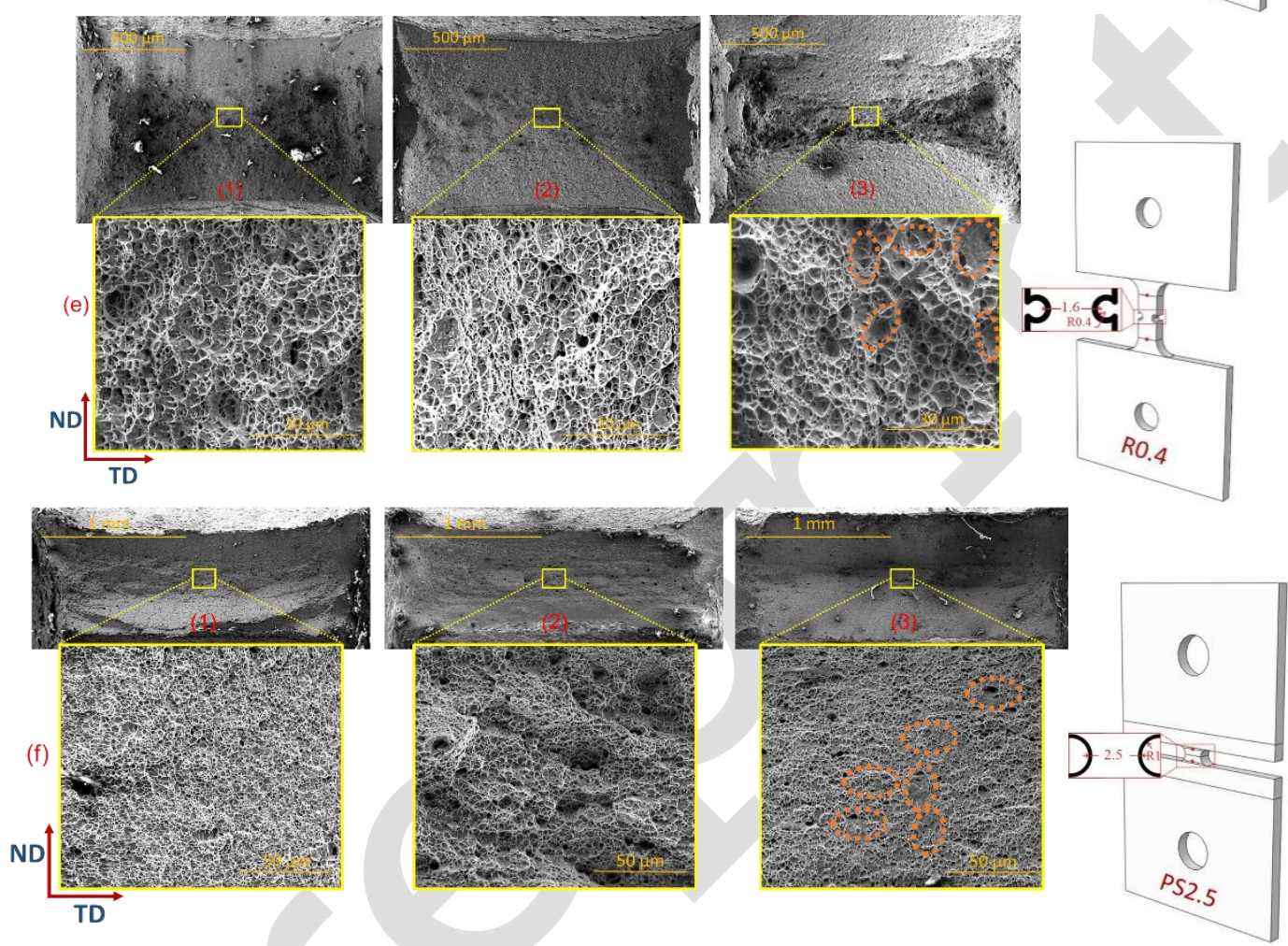

704

705
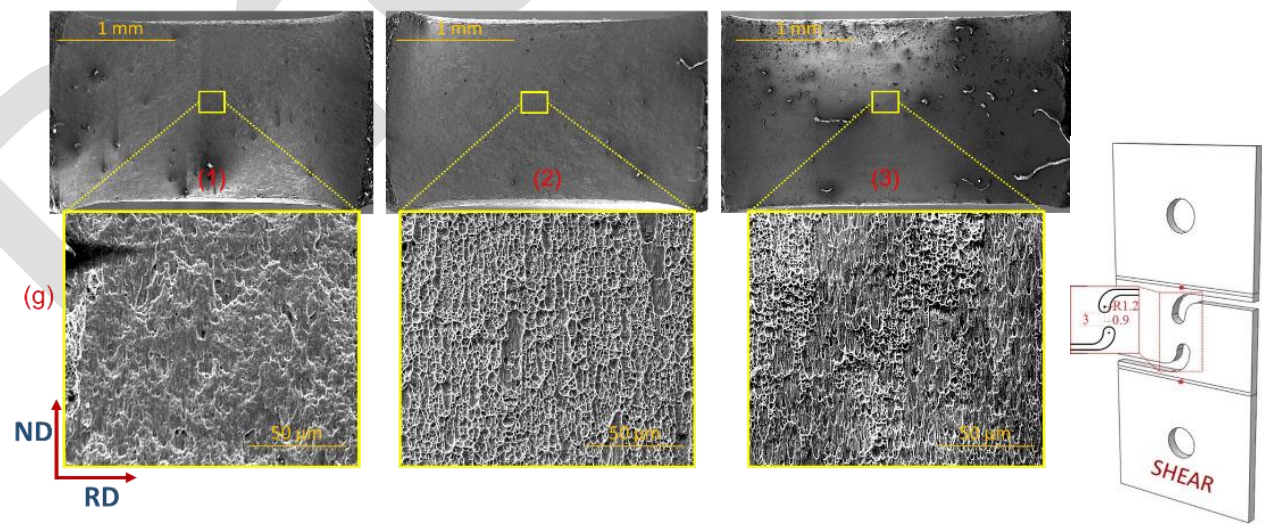

Fig 21. SEM fractographs of (a) dogbone (b) central hole, (c) R4.5, (d) R1.8, (e) R0.4, (f) PS2.5 and (g) shear

707 specimens at two magnifications. For each sample series, the left, middle and right images correspond with a static, intermediate and dynamic test, respectively. Orange ellipses display quasi-cleavage facets. 
At all the strain rates, fracture starts at the hole edge, propagates along the ligament and finally a shear lip is formed at the outer edge completing the macroscopic failure. At intermediate strain rates, micro-cracks are recognized along with the dimples. The cracks combined with the fine, uniformly distributed deep dimples result in the highest fracture strain at this rate. The most shallow dimples are again detected at dynamic strain rates, however, as opposed to the dogbone samples, no quasi-cleavage zones are observed.

The fracture surfaces of the notched R4.5, R1.8 and R0.4 specimens, presented in figs.21c, $\mathrm{d}$ and e respectively, show that an increased stress triaxiality gives rise to a more pronounced heterogeneity in the size, shape and distribution of the dimples. The smallest dimples are found for the R4.5 samples, followed by the R1.8 and R0.4 samples. Higher stress triaxialities accelerate void growth at the expense of void nucleation. At the same time, a decreasing Lode angle parameter induces a change in dimple shape. Intermediate testing speeds again result in deeper dimples and a higher dimple density. So, both an enhanced nucleation and growth of voids, gives rise to the higher fracture strain compared to the static samples. At all the strain rates, the higher the stress triaxiality, the lower the sample thinning. At dynamic strain rates, shear lips constitute a major fraction of the fracture surface. The shear lips together with sporadic quasi-cleavage facets hinder local thinning. Non-proportional loading conditions introduce mixed tensile-shear mode of fracture in these samples which is evident from the aspect ratio and orientation of dimples in the critical zone. However, the presence of special features such as serpentine glide on the dimple walls, particularly for the samples with the highest stress triaxialities, see figs.21d and e, suggests the presence of significant plasticity in DP1000 even under such unfavourable circumstances. Secondary to the central critical zone, high dimple and void densities are identified close to the notch root. Such an observation indicates that void growth and coalescence events can occur sequentially at the specimen centre and its periphery (near the root of the notch). The micro-cracks formed after the coalescence 
process at these locations thus propagate in opposing directions. These cracks eventually conjoin to effectuate macroscopic failure in R1.8 and R0.4.

Fig. 21f presents the fracture features of PS2.5 plane strain samples. As opposed to all other samples, for the PS2.5 samples, the smallest dimples are found at static rates, the largest at dynamic rates. Especially at intermediate rates, the deviation in dimple sizes becomes larger with many small dimples in between large dimples containing secondary dimples. At this rate, the dimples are also deeper, indicating a more sustained void growth before coalescence. Similar to the notched tensile samples, dimple and void fractions are dispersed along the transverse axis following the occurrence of maximum density at the centre. Hence, the subsequent void coalescence process will initiate cracks at multiple locations along the transverse mid plane. The amalgamation of these cracks thereafter results in failure. For the dynamic and static samples, the generated dimples are shallower. Quasi-cleavage is also observed in the dynamic plane strain tests, as indicated by ellipses, which further reduces the deformation capacity. Similar features are also found in PS4 samples.

The SEM fractographs of the shear fracture tests are displayed in Fig. 21g. As opposed to all other samples, the smallest dimples, even the smallest of all tests considered, are found for the static samples. Void initiation is quasi-immediately followed by coalescence, explaining the abrupt drop in force (Figs.10 e and 12a). Deeper and thus more visible dimples can be seen at the intermediate and high strain rates. The void growth lying at its origin results in a more gradual decline in load-carrying capacity at these rates (Figs. 12b and c).

For the samples dominated by tensile loading, both dimple size and shape evolve in a nonmonotonic way as a function of strain rate. Indeed, for each tensile geometry, the smallest, though deepest, and the most equiaxed dimples are found at intermediate strain rates. Consequently, not only void initiation, though also void growth is favoured at intermediate rates. Compared to static rate samples, shallow dimples with slightly higher sizes are found at 
dynamic rates. Hence, the lower fracture strains obtained at dynamic rates are mainly explained by a suppressed void growth. The shear samples deviate from the tensile samples. The smallest dimple sizes are found at static rates, the largest at intermediate rates.

Dimple size is clearly linked with stress triaxiality. For all the strain rates, in the tensiledominated samples, the smallest dimples are found for the central hole and dogbone samples, followed by the R4.5, R1.8, R0.4 samples and then the plane strain samples.

The shape of the dimples is dominated by the Lode angle. Quasi-equiaxed dimples are obtained for the central hole, R4.5 and dogbone samples. The more the Lode angle parameter evolves to 0, the more the dimples evolve to elliptical shapes. For the shear samples, strongly elongated dimples are observed for all samples. Strain rate further favours deviations from the equiaxed dimple shape, however, again in a non-monotonic way: for all the tensile dominated samples, the highest differences between the elliptical dimples axes are found at dynamic rates, the lowest at intermediate rates. For the shear samples, the highest difference is found for the dynamic samples, followed by the intermediate and static samples.

\section{Discussion}

The aforementioned results provide a comprehensive insight into the stress state and strain rate dependent plasticity and ductile fracture of the studied DP1000 steel. Irrespective of the test condition, the material exhibits a continuous yielding behaviour with excellent deformation values, in addition to excellent strength levels. Both are attributed to the ultra-fine grain microstructure. Higher deformation rates induce higher dislocation speeds which require higher stress levels to overcome the critical resolved shear stress. As a result, a positive effect of strain rate on the flow stress is obtained. Especially, at dynamic deformation rates a steeper rise of flow stress with strain rate is observed, resulting in a two-stage dependency of strength on strain rate in agreement with [Beynon et al., 2005; Oliver et al., 2007; Yu and Gao, 2009; Kim et al., 2013; Cao et al., 2015]. The significant strain hardening exhibited by the DP1000 could be due 
to the unlocking of dislocations which are introduced into the ferrite during material processing by stresses induced by the formation of martensite as described in [Cao et al., 2015].

Adiabatic heating of the material at high strain rates often counteracts the effect of strain rate. Indeed, an increased temperature decreases the dislocation resistance and induces material softening. Hence, the outcome of dynamic tests is affected by both strain rate and temperature. In the present work, a procedure is adopted to separate the temperature and strain rate effects, thus allowing to reveal the real strain rate sensitivity of the material. The procedure relies on temperature measurements performed during all tests. Furthermore, the temperature profiles allowed to calculate the fraction of plastic work contributing to a temperature rise of the material. Under dynamic testing conditions, this fraction, i.e., the Taylor-Quinney coefficient, saturates at a value of 0.88 which is in line with values mentioned in [Taylor and Quinney, 1934; Zaera et al., 2013; Roth and Mohr, 2014; Lian, 2015]. It is also worth mentioning that the temperature correction approach employed in this work is suitable for the vast majority of bcc steels.

For metals, generally, the gain in strength with rising strain rates occurs at the expense of ductility in the material. However, in the present study, a peculiar, non-monotonic dependence of the deformation capacity on the strain rate is observed in all test series and confirmed by the fracture surface analysis. Stable plastic and fracture deformation are found to increase with strain rate in the quasi-static and intermediate strain rate regime. The highest deformation values are found at intermediate strain rates, the lowest at dynamic rates. This finding challenges the generally assumed decrease of fracture strain with increasing strain rate adopted in the formulation of widely used material damage models. It also reflects the complexity associated with the investigated material. The non-monotonic evolution of the deformation might be attributed to the local temperature rise due to deformation localization at the ferrite-martensite interfaces which further improves dislocation mobility and deformation capacity. Localized 
heating, although not yet fully adiabatic at static and intermediate rates, compensates the increased, strain rate induced deformation motion resistance to such an extent, resulting in improved deformation values. At dynamic strain rates, the temperature increase, even though much more significant, no longer compensates for the increased resistance. The accompanying heat generation in the quasi-static and intermediate tests causes a temperature rise which accelerates the dislocation mobility in the ferrite. The assumed critical dependence on temperature might be further supported by the fact that DP steels are known to be prone to dynamic strain aging (DSA) phenomena, which get activated at specific temperature ranges. For DP1000 steel, the DSA effect is delayed at high strain rates [Chandran et al., 2019]. As such, it can be speculated that the temperature rise does not yet trigger the DSA phenomenon at intermediate loading speeds. However, at dynamic strain rates, the significantly higher temperature rise in the samples pushes the internal temperature closer to the DSA activation temperature thus locking further dislocation movement in the ferrite. In this case, martensite is forced to accommodate the strain incompatibilities which favours fracture at ferrite-martensite grain boundaries or in the martensite grains [Steinbrunner et al., 1988; Mazinani and Poole, 2007; Kadkhodapour et al., 2011; Landron et al., 2013; Ghadbeigi et al., 2013]. Moreover, significant strain localization in the martensite might result in dislocation clustering and entanglement which then enforces fragmentation in martensite laths with cleavage facets [Das et al., 2017; Xu et al., 2019]. This effect assists early void formation at dynamic strain rates which substantially deteriorates both strain hardening and local ductility [Steinbrunner et al., 1988; Mazinani and Poole, 2007; Kadkhodapour et al., 2011]. Analysis of the failure loci for the different strain rate regimes confirms this judgement. A non-monotonic influence of strain rate (Figs.17 and 18) on local ductility is thus established for the DP1000 steel. For quasi-static up to intermediate strain rates, increasing mobile dislocations could alter the dislocation configuration near the grain boundaries. Under this circumstance, dislocations start to pile up. 
This results in an enhanced density of dislocations at the ferrite-martensite interface. A continuous increase in the dislocation density with increasing strain rate, motivated additionally by the gradually increasing internal temperature, encourages the generation of dislocation cells in the ferrite grains. The increasing difference in the plastic deformation energy between the ferrite matrix and the martensite/ferrite interface could activate multiple slip systems in some ferrite grains. A more uniform energy distribution prevalent in such a case could actively delay the initiation and propagation of micro voids. Thus, an improvement in fracture elongation could be ascertained in these strain rate regimes. On the other hand, an accelerated generation of dislocation tangles at high strain rates does not leave room for an efficient plastic deformation energy transfer and thereby makes the material susceptible to early instabilities. It has to be mentioned that the aforementioned statements are first-order postulates that could explain the complex experimental observations made in this work. More advanced microstructural characterization is needed to fully reveal the root causes of the phenomena revealed in this research.

Next to rising deformation speeds, increasing the stress triaxiality proves to be harmful to the material ductility. Multiaxial stress states promote accelerated void growth at interfaces close to the numerous martensite grains [Ghadbeigi et al., 2013; Tasan et al., 2014; Das et al., 2017; Santos et al., 2019] which could further induce the formation of shear slip bands along martensite thereby constraining further deformation. Moreover, superior strain hardening rates under higher stress triaxiality prompt strain incompatibilities and promote faster void nucleation. The detrimental effect of stress triaxiality on the failure strain is observed over the entire range of strain rates. The fracture surfaces of the tensile-dominated samples show highly plastically deformed zones that failed perpendicular to the loading direction and shear lips aligned with inclined, localised deformation bands. All fracture surfaces are mainly covered by dimples indicating a ductile fracture mode governed by void initiation, growth and coalescence. 
Similar findings are reported in [Liu et al., 2019]. The dimple size is linked with the initiation of voids, which is clearly favoured at intermediate rates. An increase in stress triaxiality accelerates the void growth and restricts further void nucleation thus lowering local ductility. Intensification of localised shear bands, resulting in shear lips and quasi-cleavage facets under dynamic loading rates further attenuate the deformation capacity. Indeed, a significantly larger portion of the fracture surface is occupied by shear lips at dynamic deformation rates. The highly localised strain in these shear regions restricts further thinning of the sample.

The obtained 3D fracture loci suggest competing influences of stress triaxiality and Lode angle that control the course of the plane stress fracture envelope (red dotted lines in Figs. 16 and 17) between pure shear and tensile-dominated modes. An increase in stress triaxiality increases the tensile stress acting on the plane of maximum shear. The detrimental effect of the normal stress results in a higher ductility for pure shear than for uniaxial tension. The Lode angle parameter, on the contrary, points in the opposite direction. Indeed, at lower Lode angle parameters, the probability of shear localization is higher. While following the trajectory of the red dotted lines (in Figs. 16 and 17), i.e. the plane stress path, from pure shear to tension, we have $\left(\partial \varepsilon_{e q}^{p f} / \partial \eta\right) d \eta<0$ and $\left(\partial \varepsilon_{e q}^{p f} / \partial \eta\right) d \eta>0$ thus suggesting the competition between the two stress state parameters. However, from the fracture envelopes in Fig. 20a, it can be concluded that the stress triaxiality effect is stronger than the Lode angle effect for DP1000 steel. The observation of the lowest strength exhibited by the shear stress state together with the high sensitivity of the fracture strain on strain rate for samples with low Lode angle parameter suggest potential secondary Lode angle effects.

Loading histories, in terms of $\eta$ and $\bar{\theta}$, additionally play an important role in the occurrence of certain failure modes in DP1000 steel. Fracture experiments with both proportional and more complex loading paths allowed to derive the path dependence of ductile fracture. Besides establishing the relationships between imposed stress states and the underlying damage 
mechanisms, proportional loading conditions ensure that a unique, material intrinsic fracture locus is obtained which enhances the reliability of the calibration of phenomenological ductile fracture models. Non-proportional loading paths, on the other hand, are a more realistic representation of loading paths occurring in structural components. Fracture assessment under non-proportional stress states for the DP1000 reveals the presence of mixed modes of failure arising from increased strain heterogeneity in the microstructure. Associated fracture surfaces consist of non-equiaxed dimples with traces of serpentine glides on the dimple walls. Increasing stress triaxialities give rise to higher strain hardening capability, however, at the expense of ductility. However, this rise in hardening capacity is comparatively diminished under nonproportional loading paths. Similar conclusions are made based on numerical simulations and analysis [Benzerga et al., 2012; Basu and Benzerga, 2015]. Loading path changes further provoke instability prematurely at dynamic strain rates.

Irrespective of the stress state, lower strain hardening capability together with reduced deformation capacity under dynamic loading diminishes the energy absorption potential of DP1000 steel under impact loading conditions.

\section{Concluding remarks}

With the aim of elucidating the effect of strain rate on plasticity, damage and fracture properties of DP1000 steel, an extensive series of tests were carried out. A wide range of explicitly defined stress states and deformation rates were considered. The following conclusions were drawn from the research:

1. Excellent strength and deformation capacity are exhibited in all tests by the DP1000 steel by virtue of the ultra-fine grain size prevalent in the material.

2. The mechanical response of DP1000 is sensitive to both the stress state and strain rate. A positive strain rate effect on the material strength is apparent across the entire strain 
rate range and for all the stress states. A steeper rise in flow stress at dynamic loading rates revealed a two-stage relation in strain rate sensitivity. The difference in strain rate sensitivity between the two regimes is amplified by the stress triaxiality.

3. A temperature rise in the samples tested at high strain rates induces a coupled strain rate-temperature response. A correction procedure is applied to isolate thermal softening allowing to distinguish between real strain rate and temperature effects.

4. Comparison of measured and calculated temperature rises suggests that adiabatic conditions can be assumed from a strain rate of around $10 \mathrm{~s}^{-1}$ with a Taylor-Quinney coefficient of 0.88 .

5. Fracture experiments with proportional loading histories facilitate the determination of reliable material intrinsic fracture loci for DP1000 steel and its dependency on strain rate. Next to clearly showing the influence of stress triaxiality and Lode angle on the fracture strain at different strain rates, the loci can be used in ductile fracture models.

6. A rising stress triaxiality has a detrimental impact on the material's global and local ductility. Independent of the Lode angle, a monotonically decreasing fracture strain with respect to stress triaxiality is observed at all the loading rates. However, a competing effect of stress state parameters on material toughness was also evident. Dominant stress triaxiality effect is established throughout the tested range for DP1000 steel. The influence of the Lode parameter is eminent with rising strain rate.

7. Fracture experiments at varying loading speeds exposed a non-monotonic strain rate dependency on strain to failure. The low Lode angle zone displays elevated sensitivity to this strain rate induced fluctuations in fracture strain.

8. Loading path dependence on the material behaviour is perceptible particularly concerning material hardening, ductility and the resultant fracture modes. Non- 
proportional loading histories result in reduced strain hardenability, earlier onset of instability and give rise to a mixed tensile/shear mode of fracture.

9. Ductile fracture governed by void initiation, growth and coalescence was noticed in all fracture experiments. From shear to plane strain tension, the dominant failure mode transitioned from shear failure to tensile dominated fracture with shear lips. High stress triaxialities resulted in early failure as a result of accelerated void growth and coalescence process. Intensification of localized shear regions and quasi-cleavage facets under dynamic loading rates further attenuated the dimple fracture and in turn the deformation capacity.

10. Finite element simulations were used to optimise the sample geometries aiming at specific stress states interesting for damage characterization. Although the outcome of the specimen design is dependent on the material behaviour, the proposed research methodology to identify the damage behaviour of metals, and more specifically how damage is affected by stress state, stress path and strain rate, on a non-ambiguous basis, is widely applicable. Indeed, the proposed samples are easy-to-manufacture, and required test setups and measurement techniques are widely available.

\section{Acknowledgement}

The authors gratefully acknowledge the European Commission Research Fund for Coal and Steel (RFCS) for the funding of project "Toolkit for the design of damage tolerant microstructures" [grant number : 709711] which supports the investigations.

\section{References}

1. WorldAutosteel. Ultra-light steel auto body -advanced vehicle technology (ULSABAVC) programme, www.ulsab.org, overview report; 2016. 
2. J. Lian, A generalized hybrid damage mechanics model for steel sheets and heavy plates, PhD Thesis, Department of Ferrous Metallurgy, RWTH Aachen University, Aachen, Germany, 2015. Source: https://d-nb.info/1133364063/34

3. J.H. Kim, D. Kim, H.N. Han, F. Barlat, M.G. Lee, Strain rate dependent tensile behaviour of advanced high strength steels: Experiments and constitutive modelling, Material Science and Engineering A, Vol. 559 (2013), pp. 222-231. https://doi.org/10.1016/j.msea.2012.08.087

4. D. Anderson, S. Winkler, A Bardecik, M.J. Worswick, Influence of stress triaxiality and strain rate on the failure behaviour of a dual-phase DP780 steel, Materials \& Design (2014), pp. 198-207. https://doi.org/10.1016/j.matdes.2014.03.073

5. T. Rahman, A. Bardelcik, J. Imber, C. Butcher, M.J. Worswick, Effect of strain rate on flow stress and anisotropy of DP600, TRIP780 and AA5182-O sheet metal alloys. International Journal of Impact Engineering, Vol. 88 (2016), pp. 72-90. https://doi.org/10.1016/j.ijimpeng.2015.09.006

6. B. Erice, C. Roth, D. Mohr, Stress state and strain rate dependent ductile fracture of dual-phase and complex phase steel, Mechanics of Materials, Vol. 116 (2018), pp. 11 32. https://doi.org/10.1016/j.mechmat.2017.07.020

7. Y. Chen, A.H Clausen, O.S. Hopperstad, M. Langseth, Application of a split Hopkinson tension bar in a mutual assessment of experimental tests and numerical predictions, International Journal of Impact Engineering, Vol. 38 (2011), pp. 824-836. https://doi.org/10.1016/j.ijimpeng.2011.05.002

8. N.K. Singh, E. Cadoni, M.K. Singha, N.K Gupta, Dynamic tensile behaviour of multiphase high yield strength steel, Materials \& Design, Vol. 32 (2011), pp. 50915098. https://doi.org/10.1016/j.matdes.2011.06.027 
9. J. Qin, R. Chen, X. Wen, Y. Lin, M. Liang, F. Lu, Mechanical behaviour of dual-phase high strength steel under high strain rate tensile loading, Material Science and Engineering A, Vol. 586 (2013), pp. 62-70. https://doi.org/10.1016/j.msea.2013.07.091

10. N.D. Beynon, T.B. Jones \& G. Fourlaris, Effect of high strain rate deformation on microstructure of strip steels tested under dynamic tensile conditions, Material Science and Technology, Vol. 21:1 (2005), pp. 103-112. https://doi.org/10.1179/174328405X16234

11. Y. Cao, B. Karlsson, J. Ahlsström, Temperature and strain rate effects on the mechanical behaviour of dual-phase steel, Material Science and Engineering A, Vol.636 (2015), pp. 124-132. https://doi.org/10.1016/j.msea.2015.03.019

12. H. Yu, Y.G. Guo, X. Lai, Rate-dependent mechanical behaviour and constitutive model of DP600 steel at strain rate from $10^{-4}$ to $10^{3} \mathrm{~s}^{-1}$, Materials \& Design, Vol.30, Issue 7 (2009), pp. 2501-2505. https://doi.org/10.1016/j.matdes.2008.10.001

13. S. Oliver, T.B. Jones, G. Fourlaris, Dual-phase versus TRIP strip steels: Comparison of dynamic properties for automotive crash performance, Material Science and Technology, Vol. 23, Issue 4 (2007), pp. 423-431. https://doi.org/10.1179/174328407X168937

14. M. Xu, H. Li, R.T. Jiang, D. Tang, H.T. Jiang, Z.L. Mi, Deformation behaviour and microstructural evolution in ultra-high-strength dual-phase (UHS-DP1000) steel with different strain rates, Journal of Iron and Steel Research International, Vol. 26 (2019), pp. 173-181. https://doi.org/10.1007/s42243-018-0213-1

15. A. Das, M. Ghosh, S. Tarafder, S. Sivaprasad, D. Chakrabarti, Micromechanisms of deformation in dual-phase steels at high strain rates, Material Science and Engineering A, Vol. 680 (2017), pp. 249-258. https://doi.org/10.1016/j.msea.2016.10.101 
16. W. Wang, M. Li, C. He, X. Wei, D. Wang, H. Du, Experimental study on high strain rate behaviour of high strength 600-1000 MPa dual-phase steels and $1200 \mathrm{MPa}$ fully martensitic steels, Materials \& Design, Vol.47 (2013), pp. 510-521. https://doi.org/10.1016/j.matdes.2012.12.068

17. R. Alturk, S. Mates, Z. Xu, F. Abu-Farha, Effects of microstructure on the strain rate sensitivity of advanced steels, Supplemental Proceedings of $146^{\text {th }}$ Annual Meeting \& Exhibition TMS 2017, The Minerals, Metals \& Materials Series, Springer, Cham (2017), pp. 243-254. https://doi.org/10.1007/978-3-319-51493-2_24

18. Y. Bai, T. Wierzbicki, A new model of metal plasticity and fracture with pressure and Lode dependence, International Journal of Plasticity, Vol.24 (2008), pp. 1071-1096. https://doi.org/10.1016/j.ijplas.2007.09.004

19. Y. Bao, T. Wierzbicki, On fracture locus in the equivalent strain and stress triaxiality space, International Journal of Mechanical Sciences, Vol.46 (1) (2004), pp. 81-98. https://doi.org/10.1016/j.ijmecsci.2004.02.006

20. C.C. Roth, D. Mohr, Effect of strain rate on ductile fracture initiation in advanced high strength steel sheets: Experiments and modelling, International Journal of Plasticity, Vol. 56 (2014), PP. 19-44. https://doi.org/10.1016/j.ijplas.2014.01.003

21. D. Mohr, S. Henn, Calibration of stress triaxiality dependent crack formation criteria: a new hybrid experimental-numerical method, Experimental Mechanics, Vol. 47 (6) (2007), pp. 805-820. https://doi.org/10.1007/s11340-007-9039-7

22. D. Mohr, M. Doyoyo, Experimental investigation on the plasticity of hexagonal aluminium honeycomb under multiaxial loading, Journal of Applied Mechanics, Vol. 71 (3) (2004), pp. 375-385. https://doi.org/10.1115/1.1683715 
23. J. Peirs, P. Verleysen, J. Degrieck, Novel technique for static and dynamic shear testing of Ti6Al4V sheet, Experimental Mechanics, Vol.52 (7) (2012), pp. 729-741. https://doi.org/10.1007/s11340-011-9541-9

24. L. Driemeir, M. Brünig, G. Micheli, M. Alves, Experiments on stress-triaxiality dependence of material behaviour of aluminium alloys, Mechanics of Materials, Vol. 42 (2) (2010), pp. 207-217. https://doi.org/10.1016/j.mechmat.2009.11.012

25. C. Landron, O. Bouaziz, E. Maire, J. Adrien, Experimental investigation of void coalescence in a dual-phase steel using X-ray tomography, Acta Materialia, Vol.61(18) (2013), pp. 6821-6829. https://doi.org/10.1016/j.actamat.2013.07.058

26. H. Ghadbeigi, C. Pinna, S. Celotto, Failure mechanism in DP600 steel: Initiation, evolution and fracture, Material Science and Engineering A, 588 (2013), pp. 420-431. https://doi.org/10.1016/j.msea.2013.09.048

27. D.L. Steinbrunner, D.K. Matlock, G. Krauss, Void formation during tensile testing of dual-phase steels, Metallurgical transactions A, Vol.19 (1988), pp. 579-589. https://doi.org/10.1007/BF02649272

28. J. Kadkhodapour, A. Butz, S.Z. Rad, Mechanisms of void formation during tensile testing in a commercial dual-phase steel, Acta Materialia, Vol.59 (2011), pp. 25752588. https://doi.org/10.1016/j.actamat.2010.12.039

29. M. Mazinani, W.J. Poole, Effect of martensite plasticity on the deformation behaviour of a low carbon dual-phase steel, Metallurgical and Materials Transactions A, Vol.38 (2007), pp. 328-339. https://doi.org/10.1007/s11661-006-9023-3

30. T. Chiyatan, V. Uthaisangsuk, Mechanical and fracture behaviour of high strength steels under high strain rate deformation: Experiments and modelling, Material Science and Engineering A, Vol. 779 (2020), 139125. https://doi.org/10.1016/j.msea.2020.139125 
31. P. Verleysen, J. Degrieck, T. Verstraete, J. Van Slycken, Influence of specimen geometry on Split Hopkinson Tensile bar tests on sheet materials, Experimental Mechanics, Vol.48 (2008), pp.587-593. https://doi.org/10.1007/s11340-008-9149-x

32. International Organization for Standardization, Metallic materials - Tensile Testing Part 1: Methods of test at room temperature (2019) (ISO 6892-1:2019). https://www.iso.org/standard/78322.html

33. D. Novokshanov, B. Döbereiner, M. Sharaf, S. Münstermann, J. Lian, A new model for upper shelf impact toughness assessment with a computationally efficient parameter identification algorithm, Engineering Fracture Mechanics, Vol. 148 (2015), pp. 281303. https://doi.org/10.1016/j.engfracmech.2015.07.069

34. W. Liu, J. Lian, S. Münstermann, C. Zeng, X. Fang, Prediction of crack formation in the progressive folding of square tubes during dynamic axial crushing, International Journal of Mechanical Sciences, Vol. 176 (2020), 105534. https://doi.org/10.1016/j.ijmecsci.2020.105534

35. J. Lian, M. Sharaf, F. Archie, S. Münstermann, A hybrid approach for modelling of plasticity and failure behaviour of advanced high strength steel sheets, International Journal of Damage Mechanics, Vol.22 (2) (2013), pp. 188-218. https://doi.org/10.1177/1056789512439319

36. R.O. Santos, L.B. da Silveira, L.P. Moreira, M.C. Cardoso, F.R.F. da Silva, A. dos Santos Paula, D.A. Albertacci, Damage identification parameters of dual-phase 600-800 steels based on experimental void analysis and finite element simulations, Journal of Materials Research and Technology, Vol.8 (1) (2019), pp.644-659. https://doi.org/10.1016/j.jmrt.2018.04.017 
37. C.C. Roth, D. Mohr, Determining the strain to fracture for simple shear for a wide range of sheet metals, International Journal of Mechanical Sciences, 149 (2018), pp. 224-240. https://doi.org/10.1016/j.ijmecsci.2018.10.007

38. C.C. Tasan, J.P.M. Hoefnagels, M. Diehl, D.Yan, F. Roters, D. Raabe, Strain localization and damage in dual-phase steels investigated by coupled in-situ deformation experiments and crystal plasticity simulations, International Journal of Plasticity, Vol.63 (2014), pp. 198-210. https://doi.org/10.1016/j.ijplas.2014.06.004

39. International Organization for Standardization, Metallic materials: Tensile testing at high strain rates-Part1: Elastic-bar-type systems (2018) (ISO 26203-1:2018). https://www.iso.org/standard/43441.html

40. G.I. Taylor, H. Quinney, The latent energy remaining in a metal after cold working, Proceedings of Royal Society A, Vol.143 (1934), pp. 307-326. https://doi.org/10.1098/rspa.1934.0004

41. B. Wu, X. Li, Y. Di, V. Brinnel, J. Lian, S. Münstermann, Extension of modified BaiWierzbicki model for predicting ductile fracture under complex loading conditions, Fatigue and Fracture of Engineering Materials and Structures, Vol.40 (12) (2017), pp. 2152-2168. https://doi.org/10.1111/ffe.12645

42. R. Zaera, J.A. Rodríguez-Martínez, D.Rittel, On the Taylor-Quinney coefficient in dynamically phase transforming materials. Application to 304 stainless steel, International Journal of Plasticity, Vol.40 (2013), pp. 185-201. https://doi.org/10.1016/j.ijplas.2012.08.003

43. W. Liu, J. Lian, S. Münstermann, Damage mechanism analysis of a high-strength dualphase steel sheet with optimized fracture samples for various stress states and loading rates, Engineering Failure Analysis, Vol. 106 (2019), 104138. https://doi.org/10.1016/j.engfailanal.2019.08.004 


\section{Graphical Abstract}

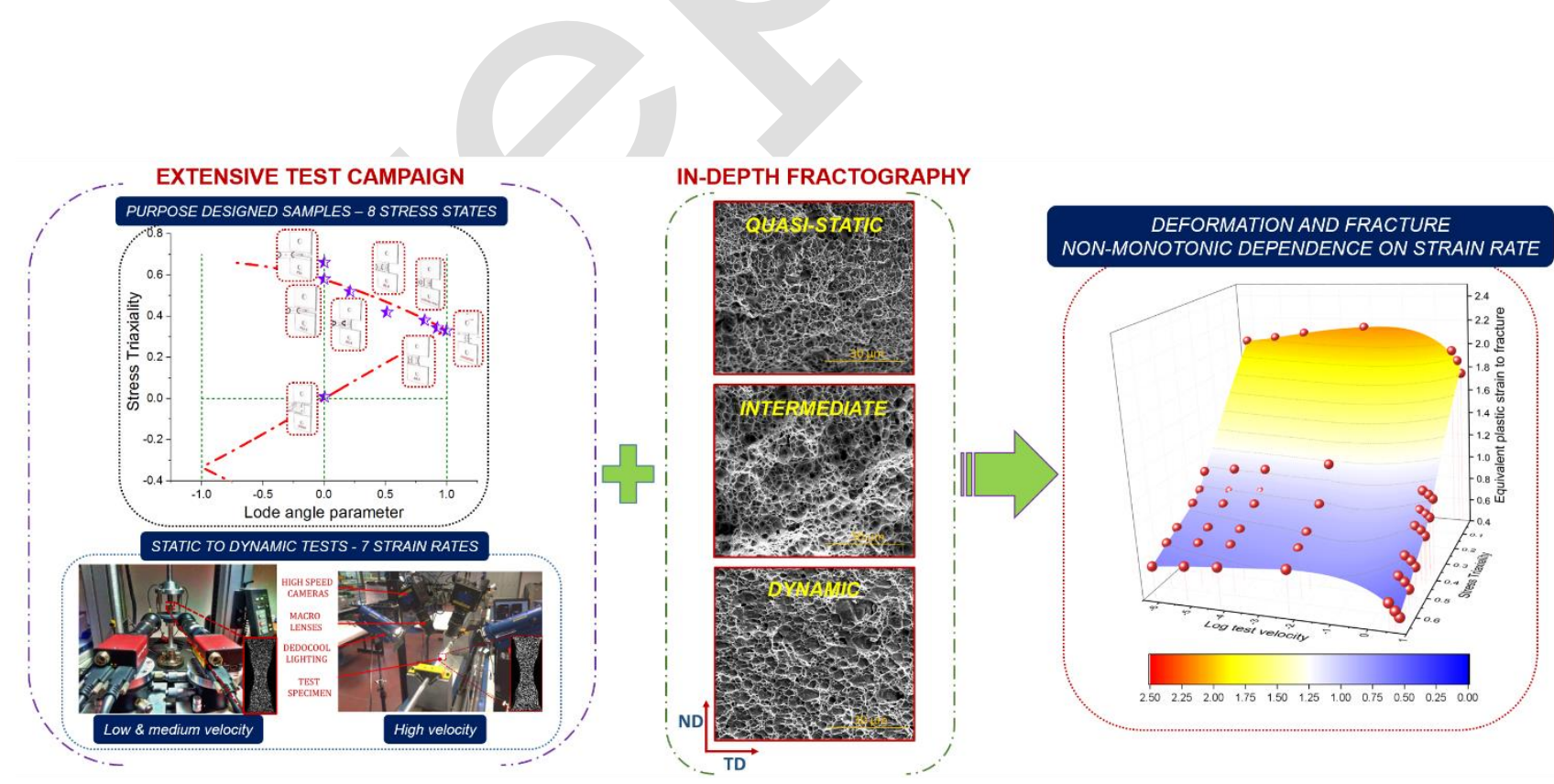

44. S. Chandran, W. Liu, J. Lian, S. Münstermann, P.Verleysen, Thermal effects on the dynamic damage and fracture of a dual-phase automotive steel, Proceedings of the $24^{\text {th }}$ DYMAT Technical Meeting: Temperature dependence of material behaviour at high strain rate, 2019, Ref: DYMAT 2019 P04.

45. S. Basu, A.A.Benzerga, On the path-dependence of the fracture locus in ductile materials: Experiments, International Journal of Solids and Structures, Vol.71 (2015), pp.79-90. https://doi.org/10.1016/j.ijsolstr.2015.06.003

46. A.A. Benzerga, D. Surovik, S.M. Keralavarma, On the path-dependence of the fracture locus in ductile materials- Analysis, International Journal of Plasticity, Vol.37 (2012), pp.157-170. https://doi.org/10.1016/j.ijplas.2012.05.003 